Abstracts from the

\title{
Abstracts from the 2016 NIH Office of Research on Women's Health BIRCWH-SCOR Meeting June 7, 2016
}




\title{
The Office of Research on Women's Health
}

\author{
2016 Meeting Report of the Signature Research \\ and Career Development Programs
}

\author{
Lisa Begg, Dr.PH, R.N., and Leah Miller, PhD, MBA
}

\section{Building Interdisciplinary Research Careers in Women's Health}

ORWH designed, developed, and implemented the Building Interdisciplinary Research Careers in Women's Health (BIRCWH) institutional career development program in 1999 to increase the number of women's health researchers working in a mentored interdisciplinary environment. BIRCWH supports junior faculty members who have recently completed clinical training or postdoctoral fellowships and are beginning basic, translational, clinical, or health services research related to women's health research by pairing them with senior investigators. BIRCWH is built around three pillars: strong mentoring, interdisciplinary research, and career development (1).

The June 2016 meeting opened with a Keynote address, entitled "Gender Equity and Success in Academic Medicine: What I Learned from Studying NIH Career Development Awardees". This was followed by a panel of the BIRCWH principal investigators discussing ways to integrate the BIRCWH and Specialized Centers of Research on Sex Differences (SCOR) programs within the same institution, entitled "Career development, Career Advancement, and Research Excellence'”. The director of the newly created NIH Division of Biomedical Research Workforce presented her plans for future training and career development for both physicians and post-doctoral researchers. Collectively, the speaker talks and panel discussion provided information to principal investigators and scholars on facing and overcoming challenges in their career including those related to career development and advancement as well as ways to exercise research excellence. The morning plenary session closed out with three (3) BIRCWH Scholars giving presentations of their research. The remainder of the day included a session on mentoring from a number of NIH institute and center staff, and the Poster session. The full list of the active BIRCWH programs can be found at: http://orwh.od.nih .gov/career/bircwh/pdf/Active-BIRCWH-Programs.pdf. And the plenary session can be viewed from the NIH videocast archive at: https://videocast.nih.gov/summary.asp?Live $=19247$ \&bhcp $=1$

\author{
Specialized Centers of Research (SCOR) \\ on Sex Differences
}

The Specialized Centers of Research (SCOR) on Sex Differences was designed and implemented in 2002 with emphasize on interdisciplinary team science to advance studies on sex differences. The SCOR program is trans-NIH (co-funded by ORWH and the National Institute on Aging, National Institute of Arthritis and Musculoskeletal and Skin Diseases, the Eunice Kennedy Shriver National Institute of Child Health and Human Development, the National Institute of Diabetes, Digestive and Kidney Diseases), and bi-agency with the U.S. Food and Drug Administration. Each SCOR site boasts an integrated basic, clinical, and translational component which explores a health issue that affects women. The current SCOR principal investigators are conducting research which explores sex and gender differences in pain, depression, vascular dysfunction and cognitive decline, substance use, tobacco dependence, musculoskeletal diseases, and health of the urinary tract health as well as reproductive health including polycystic ovary syndrome, hormonal transition, and pelvic floor disorder. The SCOR program has led to discoveries into sex differences observed in mental disorders, drug treatment and cues, fracture risk, and better understanding of the underlying mechanisms of urinary tract infections to aid in development of new therapeutic targets. In addition, the SCOR PIs have formed numerous cross-center collaborations including harmonization of measurement instruments; functional magnetic resonance imaging (fMRI) methodology approaches; and collaborative studies on brain function gender differences through utilization of neuroimaging markers (e.g., fMRI) (1). In addition, the SCOR PIs have authored numerous publications together. The SCOR program has been successful in addressing the NIH Strategic Plan for Women's Health Research goal 1 which is to "increase sex differences research in basic science studies"(2). At the June 2016 meeting the SCOR Directors met in a closed session to discuss research advances as well as challenges to considering sex as a biological variable in preclinical research and the challenges and benefits of interdisciplinary research.

The Office of Research on Women's Health (ORWH), National Institutes of Health (NIH), hosted its Annual Meetings for its two signature programs in research and career development in June 2016. Brief highlights of the meetings are provided below and are followed by the scholar abstracts presented at the meeting. 
1. Office of Research on Women's Health (2015) Report of the Advisory Committee on Research on Women's Health FY2013 - 2014: Office of Research on Women's Health and NIH Support for Research on Women's Health. Bethesda, MD: US Department of Health and Human Services, Public Health Service, National Institutes of Health. NIH Publication No. 15-7995. Available at http://orwh.od.nih.gov/about/acrwh/biennialreport/index.asp.
2. Office of Research on Women's Health, National Institutes of Health. Moving into the Future with New Dimensions and Strategies: A Vision for 2020 for Women's Health Research - Strategic Plan. NIH Publication No. 10-7606. Bethesda, MD. National Institutes of Health, 2010. Available at http://orwh.od .nih.gov/research/strategicplan/.

For further information, please contact Dr. Begg at beggl@ od.nih.gov 


\section{BIRCWH Poster Abstracts}

\section{P-1: Hormonal Response to Acute Stress by Menstrual Phase in Smokers and Nonsmokers}

Alicia Allen (presenting author), ${ }^{1}$ Sam Carlson, ${ }^{1}$ Lynn Eberly, ${ }^{2}$ Sharon Allen, ${ }^{1}$ Mustafa al'Absi, ${ }^{3}$ and Dorothy Hatsukami ${ }^{4}$

${ }^{1}$ Department of Family Medicine and Community Health,

${ }^{2}$ Division of Biostatistics, ${ }^{3}$ Department of Behavioral Medicine, and ${ }^{4}$ Department of Psychiatry, University of Minnesota

Background and Objective: The complex relationship between stress and smoking is known to vary by sex. Our understanding of the role of the menstrual cycle in this relationship is underdeveloped. Studying how the menstrual cycle influences stress response will inform the design of novel smoking cessation interventions for women. We sought to gather preliminary evidence on differences by menstrual phase and smoking status in the hormonal (e.g., progesterone, cortisol) response to acute stress.

Methods: This controlled crossover study enrolled healthy women aged 18-40 years with regular menstrual cycles. Participants completed 2 4-hour lab sessions, 1 each during the follicular and luteal menstrual phases. Each lab session consisted of a modified Trier Social Stress Task (TSST) at time 0 and blood sample collection at time $-5,5,10$, and 30 minutes. Analyses included linear mixed regression models.

Results: Participants ( $\mathrm{n}=27 ; 35 \%$ smokers) were, on average, $29.7 \pm 1.3$ years old, and more than half were nonwhite $(59 \%)$. Overall, regardless of menstrual phase, after the TSTT, hormonal increases were noted in progesterone (e.g., at 5 minutes in luteal phase: $+1.9 \mathrm{ng} / \mathrm{ml} \pm .86, P=.04)$ and decreases were noted in cortisol (e.g., at 30 minutes in luteal phase: $-26.2 \mathrm{ng} / \mathrm{ml}$ $\pm 7.03, P<.01)$. Menstrual phase and smoking status were not significantly related to any of the observed changes, however.

Conclusions: These observations suggest that the hormonal response to stress may not vary by menstrual phase or smoking status. This project was limited by a small sample size, however. Future work should explore the hormonal changes that take place during smoking cessation.

\section{P-2: Unusual Maintenance of X-Chromosome Inactivation Predisposes Female Lymphocytes to Increased Expression From the Inactive $\mathbf{X}$}

Montserrat C. Anguera (presenting author), Jianle Wang, and Camille Syrett

\section{Department of Biomedical Sciences, School of Veterinary} Medicine, University of Pennsylvania

Background and Objective: Females have a greater immunological advantage compared with men, yet they are more prone to autoimmune disorders. The basis for this sex bias lies in the $\mathrm{X}$ chromosome, which contains many immunity-related genes. Female mammals use X-chromosome inactivation (XCI) to generate a transcriptionally silent inactive $\mathrm{X}$ chromosome (Xi) enriched with heterochromatic modifications and XIST/Xist RNA, which equalizes gene expression between the sexes.
Methods: We examined the maintenance of XCI in lymphocytes from healthy females by using circulating lymphocytes from human donors and mouse models of lupus disease.

Results: We found that mature naïve $\mathrm{T}$ and $\mathrm{B}$ cells have dispersed patterns of XIST/Xist RNA, and they lack the typical heterochromatic modifications of the $\mathrm{Xi}$. In vitro activation of lymphocytes triggered the return of XIST/Xist RNA transcripts and some chromatin marks (H3K27me3, ubiquitin-H2A) to the $\mathrm{Xi}$. Lupus patients and mouse models of lupus have mis-localized patterns of Xist RNA on the Xi. Using single-cell RNA FISH, we observed biallelic expression of X-linked autoimmunity-related genes in female lymphocytes.

Conclusions: We propose that the $\mathrm{Xi}$ in female lymphocytes is predisposed to become partially reactivated and to overexpress immunity-related genes, providing the first mechanistic evidence for the enhanced immunity of females and their increased susceptibility to autoimmunity.

\section{P-3: The Co-Occurrence of ADHD and Anxiety in Adolescence: Differences by Gender}

Dara E. Babinski (presenting author), ${ }^{1}$ Daniel A. Waschbusch, ${ }^{1}$ James G. Waxmonsky, ${ }^{1}$ and Douglas L. Leslie ${ }^{2}$

${ }^{1}$ Department of Psychiatry and ${ }^{2}$ Department of Public Health Sciences, College of Medicine, Pennsylvania State University

Background and Objective: Although nearly 25\% of children with attention-deficit/hyperactivity disorder (ADHD) are diagnosed with an anxiety disorder, very little is known about anxiety in girls with ADHD, particularly during adolescence, when anxiety emerges substantially more among girls than boys. This study examined effects of gender and ADHD on the severity of anxiety in adolescents. Differential associations among ADHD, anxiety, and daily life impairments by gender were also explored.

Methods: Data were used from the Multimodal Treatment of ADHD (MTA) Study, which included 579 adolescents with ADHD and a comparison group of 289 adolescents without diagnosed ADHD. In adolescence, anxiety was assessed using self-reports on the Multidimensional Anxiety Scale for Children, ADHD severity was assessed by parents and teachers using the SNAP Questionnaire, and parent-reported impairment was measured by the Columbia Impairment Scale.

Results: Girls' ratings of anxiety $(\mathrm{M}=87.25, \mathrm{SD}=18.23)$ were significantly greater than boys' ratings regardless of ADHD status $(\mathrm{M}=80.24, \mathrm{SD}=29.54 ; P<.01)$. For boys, ADHD $(B=.70, P<.01)$ and anxiety severity $(B=.08, P<.05)$ explained unique variance in impairment, but only ADHD severity $(B=.74, P<.01)$ significantly explained variance in parent-rated impairment among females. These findings remained significant even when controlling for the severity of oppositional defiant behavior.

Conclusions: This work suggests that gender and developmental stage are important considerations in clarifying the nature of co-occurring ADHD and anxiety. Future work examining the life course of ADHD in girls is greatly needed. 


\section{P-4: Phenome-Wide Association Studies Identify a New Association of Atrial Fibrillation in Males with Systemic Lupus Erythematosus}

April Barnado (presenting author), ${ }^{1}$ Robert Carroll, ${ }^{2}$ Carolyn Casey, ${ }^{1}$ Josh C. Denny, ${ }^{1,2}$ and Leslie J. Crofford ${ }^{1}$

${ }^{1}$ Department of Medicine and ${ }^{2}$ Department of Biomedical Informatics, Vanderbilt University

Background and Objective: Systemic lupus erythematosus (SLE) is an autoimmune disease with a female-to-male ratio of 9:1. Although SLE is more prevalent in females, males with SLE may have increased disease severity and mortality, but they are understudied. Phenome-wide association studies (PheWAS) are a systematic and efficient approach to identifying novel clinical associations within subgroups of patients. We sought to assess differences in comorbidities between males and females with SLE by performing the first electronic health record (EHR)-based PheWAS to compare ICD-9 code-based phenotypes in males and females.

Methods: SLE cases were identified in the EHR using our validated algorithm. A PheWAS compared males and females while adjusting for covariates in a logistic model and correcting for multiple testing using the Bonferroni method.

Results: We identified 986 females and 111 males with SLE. Adjusting for race/ethnicity and current age, males were more likely than females to have cardiovascular ICD-9 codes, including atrial fibrillation (odds ratio $[\mathrm{OR}]=4.50,95 \% \mathrm{CI}, 2.32$, $\left.8.72 ; P=8.6 \times 10^{-6}\right)$; other chronic ischemic heart disease $\left(\mathrm{OR}=4.40[2.24,8.64] ; P=1.7 \times 10^{-5}\right)$; and atrial fibrillation and flutter, $\left(\mathrm{OR}=4.08\right.$ [2.12, 7.83]; $\left.P=2.4 \times 10^{-5}\right)$.

Conclusions: We report the first association of atrial fibrillation in males with SLE. While there is a five-fold increased risk of cardiovascular disease overall in SLE, the risk of atrial fibrillation, specifically in males, has not been identified. These findings demonstrate the ability of PheWAS to uncover novel phenotype associations within subgroups of a disease.

\section{P-5: Assessing for Gender Differences in the Walking Patterns of Transfemoral Amputees by Pelvic Accelerometry Tracking}

Gerasimos Bastas (presenting author)

Department of Physical Medicine and Rehabilitation, School of Medicine, Vanderbilt University

Background and Objective: Known gender-based differences in whole-body movements suggest that men and women use different neuromuscular control strategies during walking, athletic activities, and negotiating stairs. Such differences are primarily identified in the biomechanics of the pelvis and hip. This study's objective was to investigate the effect of gender on the performance of transfemoral amputees (TFAs) in walking with a prosthesis.

Methods: We performed pelvic accelerometry tracking with an inertial measurement unit placed over the fifth lumbar vertebra in 5 female and 6 male TFAs and in gender-matched controls. Analyses included within- and between-gender comparisons of estimated gait metrics.

Results: Male and female TFAs exhibited similar selfselected speeds. Female TFAs had a greater inter-limb offset for timing of acceleration/deceleration events (vs. gender-matched controls $[P=.04])$ and exhibited delayed attainment of maximal braking force (deceleration) on their sound limb, compared with the male TFAs (at $16 \%$ of the gait cycle vs. $10 \%$ for men $[P=.017]$ and $10 \%$ for female controls $[P=.03])$.

Conclusions: Pelvic accelerometry suggests that current prosthetics afford similar walking profiles for female and male TFAs. The delay of female TFAs in the attainment of the maximal braking force suggests a possible gender difference in the coordination of the weight transfer event from prosthetic to sound limb. Furthermore, their significantly skewed inter-limb offset for acceleration/deceleration events may have broader implications for socket design (stability) and reaction to a prosthetic component.

\section{P-6: Implications of Racial Discrimination for Health Among Adolescent Females on Probation}

Aerika Brittian (presenting author), ${ }^{1}$ Geri Donenberg, ${ }^{2}$ and Erin Emerson ${ }^{2}$

${ }^{1}$ Department of Educational Psychology and ${ }^{2}$ School of Public Health, University of Illinois at Chicago

Background and Objective: Racial discrimination poses notable risks to health and development for girls of color. It is well documented that girls of color are not only disproportionately afflicted by mental health disparities; they are also overrepresented in the juvenile justice system. However, few studies have examined implications of racial stress for health among female youth on probation. The objectives of this mixed-methods study were to: 1) investigate the prevalence of female youth's experiences with racial discrimination; 2) assess the implications of racial discrimination for mental health and externalizing behaviors; and 3) explore how resilience may occur among this population through the use of cultural resources and coping abilities.

Methods: Participants for this study included 65 females (aged 13-18 years; 93\% African American) who participated in a federally funded randomized controlled trial testing the efficacy of an HIV/AIDS/STI, mental health, and substance use program.

Results: Qualitative findings elucidated the most prevalent racial experiences (e.g., People acted as if they were afraid of you). Regression analyses indicated that racial discrimination was associated with increased internalizing of symptoms and externalizing behaviors, but these effects were not moderated by ethnic identity or coping abilities.

Conclusions: Contrary to prior research in community samples, although discrimination was identified as a notable risk factor for female youth's internalizing of symptoms and externalizing behaviors, none of the hypothesized factors were found to buffer the effects of discrimination on health outcomes. Therefore, our future research will focus on identifying modifiable characteristics that promote resilience among girls of color involved in the juvenile justice system, in order to reduce health disparities among this population.

\section{P-7: Lipoprotein(a) Is Associated with Subclinical Atherosclerosis in HIV-Infected Young Women}

Enkhmaa Byambaa (presenting author)

Department of Internal Medicine, Medical Center, University of California, Davis

Background and Objective: Lipoprotein(a) (Lp[a]), is an independent causal risk factor for cardiovascular disease (CVD), where levels are regulated by the apolipoprotein(a) (apo[a]) 
gene. HIV-infected individuals are at significantly elevated CVD risk compared with HIV-uninfected individuals. The role of Lp(a) in HIV-related CVD remains unclear. We investigated the association between $\mathrm{Lp}$ (a) level, apo(a) size polymorphism, and subclinical atherosclerosis in HIV-infected and HIV-uninfected women in the Women's Interagency HIV Study.

Methods: Lp(a) level, apo(a) sizes, and carotid artery intimamedia thickness (cIMT) were determined in $150 \mathrm{HIV}$-infected and $100 \mathrm{HIV}$-uninfected women. $\mathrm{Lp}$ (a) levels were square-root transformed to achieve normality. Linear regression models were used to evaluate the association of Lp(a) with cIMT, adjusting for confounders.

Results: The mean ages for the HIV+ and HIV- groups were $31 \pm 4$ and $30 \pm 4$ years, respectively. The majority of women were African American (69\% and 76\% in the HIV+ and HIV- group, respectively). HDL-cholesterol, triglyceride, and Lp(a) levels were significantly lower in the HIV+ group than in the HIVgroup. Prevalence of atherogenic small size apo(a) ( $<22$ kringles) was $\sim 20 \%$ in both groups. Notably, Lp(a) level was predictive of cIMT in the HIV+ group $(P=.029)$, but not in the HIV- group $(P=.584)$. After accounting for confounders (age, race, smoking, BMI, blood pressure, HCV co-infection, menopause, blood lipids, treatment status, CD4+ T-cell count, and HIV/RNA viral load), the association remained significant $(P=.049)$.

Conclusions: $L p(a)$ level is associated with subclinical atherosclerosis in young HIV-infected women. Further research is needed to identify mechanisms underlying Lp(a)-associated increased CVD risk in HIV-infected individuals.

\section{P-8: Sex Differences in Morphometry of the Anatomical Structures of the First Carpometacarpal Joint}

Abhijit J. Chaudhari (presenting author), ${ }^{1}$ Brent H. Foster, ${ }^{2}$ Dylan A. Noblett, ${ }^{1}$ Robert D. Boutin, ${ }^{1}$ Robert M. Szabo, ${ }^{3}$ Christopher O. Bayne, ${ }^{3}$ Richard M. Leahy, ${ }^{5}$ Nancy E. Lane, ${ }^{4}$ and Anand A. Joshi ${ }^{5}$

${ }^{1}$ Department of Radiology, ${ }^{2}$ Department of Biomedical Engineering, ${ }^{3}$ Department of Orthopedic Surgery, and ${ }^{4}$ Department of Medicine, School of Medicine, University of California, Davis; ${ }^{5}$ Signal and Image Processing Institute, University of Southern California

Background and Objective: First carpometacarpal (CMC) joint osteoarthritis $(\mathrm{OA})$ is more prevalent in women than men. We hypothesized that the sexes will exhibit morphometric differences in the anatomical structures of the first CMC joint. Knowledge of these differences will assist in understanding first CMC OA pathogenesis for each sex and inform strategies for its clinical management. We wanted to create an MRI protocol for evaluating the tissues of the first CMC joint and to develop computational tools for characterizing tissue morphometric variation by sex.

Methods: We acquired high-resolution, 3D MRIs of the first $\mathrm{CMC}$ joint tissues. We developed methods to delineate the first $\mathrm{CMC}$ joint tissues and to compare their morphology based on sex. Next, we evaluated 6 healthy women, 6 healthy agematched men, and 4 women with first CMC OA.

Results: Healthy women had an overall lower cartilage volume $\left(\right.$ median $\left.=103.3 \mathrm{~mm}^{3}\right)$ compared with healthy men $($ median $=$ $125.1 \mathrm{~mm}^{3}$ ), while the volumetric subluxation of the first metacarpal bone was higher in healthy women $($ median $=2.1 \mathrm{~mm})$ compared with healthy men (median $=1.6 \mathrm{~mm}$ ). In women with first CMC OA, the cartilage volume was lower (median= $38.8 \mathrm{~mm}^{3}$ ) and volumetric subluxation higher (median $=2.7 \mathrm{~mm}$ ), compared with both healthy women and healthy men.
Conclusions: In this pilot study the proposed methods appear to provide high sensitivity for detecting sex-specific morphometric differences in the tissues of the first CMC joint. We will continue to assess the role of these differences for sex-specific OA risk assessment and for developing sex-specific conservative/ surgical treatment algorithms.

\section{P-9: Pharmacokinetics of Lamotrigine Across Pregnancy: Considerations for Treatment of Women with Bipolar Disorder}

Crystal T. Clark (presenting author), ${ }^{1}$ Katherine L. Wisner, ${ }^{1}$ Catherine Stika, ${ }^{2}$ Stephanie Schuette, ${ }^{1}$ and Michael J. Avram ${ }^{3}$

${ }^{1}$ Department of Psychiatry, ${ }^{2}$ Department of Obstetrics and Gynecology, and ${ }^{3}$ Department of Anesthesiology, Feinberg School of Medicine, Northwestern University

Background and Objective: Lamotrigine is used for the maintenance treatment of bipolar disorder. Decreased serial ratios of lamotrigine serum concentration to dose across pregnancy compared with nonpregnant lamotrigine levels suggest that lamotrigine concentration decreases throughout pregnancy. We sought to characterize changes in lamotrigine clearance and total body volume across pregnancy in women with bipolar disorder.

Methods: After obtaining IRB-approved written informed consent, 3 pregnant women with bipolar disorder were admitted to a clinical research unit 2 to 3 times during their pregnancies and, when possible, at least once within 3 months postpartum for pharmacokinetic studies. Fifteen blood samples were obtained, including 1 prior to the patient's lamotrigine dose and 14 between 1 and 24 hours after drug administration. Plasma drug concentrations were measured by liquid chromatography-tandem mass spectrometry and were modeled with 2-compartment pharmacokinetic models.

Results: Elimination clearance and volume of distribution both increased across pregnancy, but at different rates. In the first $15 \pm 4$ (mean \pm SD) weeks of pregnancy, elimination clearance increased $65 \% \pm 29 \%$, while the volume of distribution increased only $11 \% \pm 7 \%$. By $28 \pm 5$ weeks of pregnancy, elimination clearance increased $103 \% \pm 46 \%$, while the volume of distribution increased just $38 \% \pm 8 \%$.

Conclusions: The increase in volume of distribution throughout pregnancy was only a fraction of the increase in elimination clearance. Dosing simulations suggest that increases in the dose of lamotrigine are required to accommodate the changes in lamotrigine concentration because of changes in elimination clearance throughout pregnancy.

\section{P-10: Connecting Appalachians to Lung Cancer Screening: Leveraging Social Networks to Reduce Lung Cancer Disparities}

Katherine S. Eddens (presenting author), ${ }^{1}$ Timothy Mullett, ${ }^{2}$ Jamie Studts, ${ }^{3}$ Nancy Schoenberg, ${ }^{3}$ and Li Chen ${ }^{4}$

\section{${ }^{1}$ Department of Health Behavior, College of Public Health; ${ }^{2}$ Division of Cardiothoracic Surgery; ${ }^{3}$ Department of Behavioral Science, College of Medicine; and ${ }^{4}$ Division of Cancer Biostatistics, Markey Cancer Center, University of Kentucky}

Background and Objective: Appalachian Kentucky counties have the highest rates of lung cancer incidence and mortality 
among women in the United States. Annual screening for lung cancer is recommended for eligible high-risk adults following shared decision making with a health care provider. Appalachian women are less likely than non-Appalachians to be screened for breast, cervical, and colorectal cancer within recommended guidelines, and Appalachians rely on strong kin and community networks for trusted information. This study examines the influence of communication networks on intention to screen for lung cancer in an Appalachian population that meets eligibility criteria for lung cancer screening (LCS).

Methods: Using network data collection software created by Dr. Eddens (OpenEddi), we will recruit 400 individuals from Appalachian communities and (1) assess the feasibility of using OpenEddi in this population; (2) assess knowledge, attitudes, and beliefs about LCS; (3) describe communication and social support networks of LCS-eligible individuals; (4) build a predictive model of LCS intention for high-risk Appalachians; and (5) examine sex differences in these intentions and predictors.

Results: Preliminary results among Appalachian women showed that these women find OpenEddi easy and fun to use, and the study found small, dense networks predominantly consisting of ties with kin. Participants reported better understanding of the composition and use of their communication networks when they had viewed a graph of their network.

Conclusions: Using OpenEddi is feasible in a population of rural Appalachian women, and visualizing personal communication networks helps women understand social network structure. Results of this study will inform effective communication network-based translational interventions to promote shared decision-making regarding LCS.

\section{P-11: A Community Engaged Approach to Reducing Smoking in Women's Residential Treatment Facilities for Substance Use Disorder}

Amanda Fallin (presenting author), Alana Miller, Kristin Ashford, and Ellen J. Hahn

College of Nursing, University of Kentucky

Background and Objective: Smoking prevalence is high (77\% to $93 \%$ ) among individuals with substance use disorders (SUDs), but those who stop smoking are more likely to report long-term recovery from drug or alcohol addiction. Also, pregnant smokers with SUDs are at high risk for negative maternal and infant outcomes. This study explored facilitators and barriers to smoking cessation among pregnant and parenting women with SUDs, with the intent of informing future tailored interventions.

Methods: Four clients at an SUD residential treatment facility for pregnant and parenting women participated as members of community research teams to engage the community using photovoice and social determinants mapping and assisting with leading focus groups. Next, focus groups with clients $(n=42)$ and individual interviews with staff $(n=10)$ were conducted. Focus groups and individual interviews were voice recorded, transcribed verbatim, and content analyzed using MAXQDA.

Results: Three themes emerged from client focus groups: (1) desire to quit smoking, (2) experience with periodic nicotine abstinence (e.g., while in prison), and (3) lack of confidence managing stress and other drug addiction without smoking. Staff reported that a tobacco-free policy and offering tobacco treatment would (1) help women remain tobacco free after periods of abstinence and (2) improve grounds safety. Three major barriers were (1) tobacco use as a coping strategy, (2) smoking cessation as a stressor, (3) and infringing on clients' personal choice.
Conclusions: Most clients wanted to stop using tobacco, but they lacked confidence. Despite barriers to incorporating tobacco treatment and policy, staff members were generally positive. Tailored tobacco treatment approaches for women's residential SUD treatment facilities are being explored.

\section{P-12: Perceptions of Barriers and Benefits to Adolescent Pre-Exposure Prophylaxis (PrEP) Utilization and Clinical Trial Participation: A Study of African American Mothers and Their Daughters}

Faith E. Fletcher (presenting author), ${ }^{1}$ Celia Fisher, ${ }^{4}$ Brenikki Floyd, ${ }^{1}$ Angelica Ehioba, ${ }^{1}$ Meredith Buchberg, ${ }^{5}$ and Geri Donenberg 2,3

${ }^{1}$ Division of Community Health Sciences and ${ }^{2}$ Community Outreach Intervention Projects and Healthy Youths Program, School of Public Health, and ${ }^{3}$ Department of Medicine, University of Illinois at Chicago; ${ }^{4}$ Department of Psychology, Center for Ethics Education, HIV Prevention Research Ethics Training Institute, Fordham University; ${ }^{5}$ Department of Periodontics, Health Science Center at San Antonio, University of Texas

Background and Objective: Pre-exposure prophylaxis $(\mathrm{PrEP})$ is a recently developed prevention strategy that involves the administration of Truvada to HIV-negative individuals who present high exposure or susceptibility to HIV. Currently, PrEP is not approved for adolescents, and data on adolescents and PrEP are lacking. Thus, PrEP's potential as an HIV prevention strategy for adolescent populations is unknown. Given the social, behavioral, and ethical issues surrounding PrEP use for adolescents, the purpose of this study was to assess perceptions of barriers and benefits related to African American girls' (aged 14-17 years) participation in PrEP clinical trials and potential utilization of various modes of PrEP delivery.

Methods: Fifteen African American mother/daughter pairs $(\mathrm{N}=30)$ were recruited from a 2-armed, family-based randomized controlled trial to participate in a focus group discussion and questionnaire related to adolescents' PrEP utilization and clinical trial participation. The focus group discussion was guided by a 15-minute PrEP educational module, which included a hypothetical clinical trial informed by 2 local community advisory boards.

Results: Most mothers expressed a willingness to allow their daughters to take PrEP once approved for adolescents, but they indicated concerns about clinical trial participation, primarily because of a lack of available evidence related to the long-term side effects of PrEP for adolescents. Conversely, daughters expressed a willingness to participate in PrEP clinical trials, citing both individual and society-level benefits.

Conclusions: Study findings uncover the need to implement ethically sound strategies to strengthen the availability, accessibility, and acceptability of new scientific advances in HIV for HIV-vulnerable adolescent populations.

\section{P-13: Nociceptive Neurons Differentially Drive Type I and Type II Allergic Airway Inflammation}

Simmie Foster, ${ }^{1,3}$ Sebastien Talbot, ${ }^{2,4}$ and Clifford Woolf ${ }^{2,4}$

${ }^{1}$ Department of Psychiatry and ${ }^{2}$ Department of Neurobiology, Harvard Medical School, Harvard University; ${ }^{3}$ Depression Clinical Research Program, Massachusetts General Hospital; ${ }^{4}$ F.M. Kirby Neurobiology Center, Boston Children's Hospital 
Background and Objective: Asthma is an immunological disorder characterized by mucous production, airway hypersensitivity, and inflammation. Lifetime prevalence of asthma is greater in women, and adult women are twice as likely as men to develop asthma. Intriguingly, recent evidence points to nociceptors as major drivers of many inflammatory diseases, including asthma. Immune responses may be broadly divided into type 1 responses against viruses and bacterial pathogens and type 2 responses, including allergies and asthma; women tend to have increased type 2 immunity at baseline. In this study we asked if nociceptor activity contributes differently to type 1 versus type 2 airway inflammation.

Methods: To answer our research question, we first established mouse models of type 1 or type 2 allergic airway inflammation in wild-type mice or nociceptor-ablated mice. In addition, we pharmacologically silenced nociceptors in wild-type mice with established airway inflammation. We then measured immune cell recruitment and antibody production in the different treatment groups.

Results: As we have shown previously, ablating nociceptors in classic type 2 airway disease reduced inflammatory infiltrate and airway remodeling. In contrast, we found that silencing or ablating nociceptors in a type 1 model had no effect on inflammation.

Conclusions: Our results indicate that nociceptors may preferentially participate in type 2 inflammation in the lung. Future studies will examine specifically the contribution of nociceptors to type 1 and type 2 immunity in males and females, and they will address the issue of whether excessive nociceptor drive contributes to the greater prevalence of inflammatory disease in females. We expect 1) wild-type female mice to have more severe type 2 disease than wild-type males (whereas type 1 disease may have a more even sex distribution), and 2) nociceptor ablation to reduce disease severity more in females than males.

\section{P-14: Evaluating the Repertoire of Immune Checkpoint Markers Expressed Within Ovarian Cancers}

Stéphanie Gaillard (presenting author), ${ }^{1}$ John $\mathrm{Yi}^{2}{ }^{2}$ Chelsae Dumbauld, ${ }^{2}$ Jessie Ehrisman, ${ }^{3}$ Andrew Berchuck, ${ }^{3}$ and Kent Weinhold ${ }^{2}$

${ }^{1}$ Department of Medicine, ${ }^{2}$ Department of Surgery, and ${ }^{3}$ Department of Obstetrics and Gynecology, Medical Center, Duke University

Background and Objective: Multiple costimulatory and inhibitory interactions regulate tumor-directed T-cell responses. Identification of immune-suppressive checkpoints, such as PD1/PD-L1 and CTLA-4, has led to the development of a new drug class of immune checkpoint inhibitors. The repertoire of immune checkpoint markers expressed within ovarian cancer is not known. This information is essential to identifying potential targets for immune checkpoint-directed therapy in ovarian cancer. We sought to quantify the expression of immune checkpoint markers in ascites and peripheral blood (PB) from patients with ovarian cancer.

Methods: Paired PB and ascites samples were collected from patients with epithelial ovarian cancer $(n=8)$. Cells isolated from $\mathrm{PB}$ and ascites were used for immune profiling of 9 checkpoint cell surface markers on CD4+ and CD8+ T cells by multiparameter flow cytometry. In parallel, 14 soluble check- point markers were quantitated in plasma and ascites fluid, using an Affymetrix ProcartaPlex Human Immuno-oncology checkpoint panel.

Results: In 15 of the 21 markers evaluated, expression was higher in ascites than in paired PB. For example, 50\%-92\% of CD4+ T-cells from ascites expressed PD-1, compared with $13 \%-47 \%$ of CD4+ T cells from PB. Similarly, Tim-3 was expressed on $1.1 \%-25 \%$ of CD4+ T cells in ascites, compared with $0.95 \%-2.5 \%$ of $\mathrm{CD} 4+\mathrm{T}$ cells in $\mathrm{PB}$. Trends were similar for soluble checkpoint molecules.

Conclusions: There is significant variability in the expression of immune checkpoint modulators within ovarian cancers, but immune cells within ovarian cancer-associated ascites typically express much higher levels of immune checkpoint markers than paired peripheral $\mathrm{T}$ cells. These markers may be effective therapeutic targets for ovarian cancer.

\section{P-15: Angiogenic Factors Predict Placental Weight and Birth Weight in Bangladeshi Pregnancies}

Alison D. Gernand (presenting author), ${ }^{1}$ Abdullah Al Mahmud, ${ }^{2}$ Eszter Papp, ${ }^{3}$ Joy Shi, ${ }^{3}$ and Daniel E. Roth ${ }^{3,4,5}$

${ }^{1}$ Department of Nutritional Sciences, Pennsylvania State University; ${ }^{2}$ Center for Child and Adolescent Health, International Center for Diarrhoeal Disease Research, Dhaka, Bangladesh; ${ }^{3}$ Center for Global Child Health and ${ }^{4}$ Department of Pediatrics, Hospital for Sick Children, Toronto, Ontario; ${ }^{5}$ Departments of Nutritional Sciences and Pediatrics, University of Toronto, Toronto, Ontario

Background and Objective: Angiogenic proteins are produced by the placenta and are essential for vascular development. Their concentrations in maternal blood during pregnancy have been associated with preeclampsia. Little is known about these factors in pregnancies in low-income countries or their relationship to placental and fetal growth. In Bangladesh, a setting with high rates of fetal growth restriction, we examined associations between biomarkers of angiogenesis and placental and infant weight at birth.

Methods: In an ongoing trial that enrolled pregnant women at 17-24 weeks' gestation in Dhaka, we measured 10 angiogenic biomarkers in maternal plasma collected at 30 weeks' gestation $(n=367)$. Biomarkers included vascular endothelial growth factor, placental growth factor (PIGF), angiopoietin-2, fibroblast growth factor-2, and endoglin. Characteristics of the mothers were collected at enrollment, and placentas and infants were measured at delivery.

Results: Mean (standard deviation) placental and birth weight were 368 (69) grams and 2,741 (353) grams, respectively. Twenty-two percent of infants were low birth weight $(<2,500$ grams). PIGF and angiopoietin-2 were each positively associated with placental weight $(P=.001$ and .040 , respectively), and endoglin was negatively associated with placental weight $(P=.018)$, controlling for other angiogenic factors; infant sex; and maternal age, education, and height. PlGF was positively and endoglin was negatively associated with birth weight (each at $P \leq .002$ ) after adjusting for confounders. There was no interaction by fetal sex.

Conclusions: Key angiogenic proteins at 30 weeks' gestation were predictive of placental and infant weight at birth. These biomarkers should be further explored as markers of placental development and pregnancy outcomes. 


\section{P-16: Impact of Short-Term and Continuous Estradiol Exposure Following Cessation of Ovarian Function on Arterial Remodeling in the Hippocampus}

Elin Grissom (presenting author) ${ }^{1}$ and Sarah Lindsey ${ }^{2}$

${ }^{1}$ Department of Psychology and Program in Neuroscience and ${ }^{2}$ Department of Pharmacology, Tulane University

Background and Objective: Estradiol is neuroprotective for cerebral arteries, maintaining the flexibility of vessels to allow for adequate blood flow. Restricted blood flow in the hippocampus due to thickening of artery walls and stiffening of vessels has been linked to cognitive impairments, particularly in postmenopausal women. Investigations of postmenopausal estrogen replacement, or hormone therapy, have found that the timing and duration of hormone therapy affected whether the effects of estrogen on cognitive and cardiovascular function were positive or negative. Given the mixed results of previous studies, the objective of the current project was to assess the impact of short-term and continuous estradiol replacement on arterial wall thickness and disruption of surrounding neural tissue in the hippocampus.

Methods: Aging female rats were ovariectomized and divided into 3 groups: a vehicle control group, a short-term estradiol group that received 40 days of estradiol exposure followed by 40 days with no estradiol, and a continuously exposed group that received estradiol throughout the experiment. Arteries in the hippocampus were visualized using a hematoxylin and eosin stain, and vessel wall thickness and disruption of surrounding neuropil were measured as indicators of arteriolosclerosis.

Results: Rats in the control group had greater indicators of hippocampal arterial remodeling and a higher number of arteries with indicators of severe arteriolosclerosis compared with both the short-term and continuous estradiol groups.

Conclusions: These findings indicate that estradiol likely serves to protect arteries in the hippocampus after the cessation of ovarian function and that a short course of estradiol replacement is sufficient to elicit these beneficial effects.

\section{P-17: Pubertal Timing as a Moderator of the Effects of Discrimination Experiences on Disruptive Behavior of Adolescent Girls}

Colleen A. Halliday-Boykins (presenting author) ${ }^{1}$ and Sandra Graham ${ }^{2}$

${ }^{1}$ Department of Psychiatry and Behavioral Sciences, Medical University of South Carolina; ${ }^{2}$ Department of Education, University of California, Los Angeles

Background and Objective: Understanding the effects of racial discrimination on adolescent girls' disruptive behavior requires an understanding of the unique ways that racial discrimination operates for girls. Gendered forms of racial discrimination have the potential to explain variation in adolescent girls' behavior beyond general racial discrimination. Further, because how adolescents are treated and how they react to others depends in part upon their social, cognitive, and physical development, the effects of discrimination likely vary based on physical maturation. Girls experiencing puberty early may be particularly vulnerable to social and contextual stressors, such as experiences with discrimination. The purpose of this investiga- tion was to examine the extent to which early pubertal development moderated the effects of gender, racial, and of gendered racial discrimination on girls' disruptive behavior.

Methods: Participants were 922 girls (43.0\% Latina, $27.3 \%$ Black/African American, 8.6\% White/Caucasian, 7.7\% Asian American/Pacific Islander) recruited during $6^{\text {th }}$ grade and followed through $12^{\text {th }}$ grade. Data were gathered from self-reports for pubertal development, disruptive behavior, and experiences with discrimination. Aggressive behavior was assessed via teacher reports. Data were analyzed using multilevel growth curve analyses.

Results: Early puberty magnified the adverse effects of racial discrimination and of gendered racial discrimination on selfreported conduct problems over time. Both racial and gendered racial discrimination were associated with teacher-reported aggression, but these relationships were not moderated by pubertal timing.

Conclusions: Girls who mature early might be especially vulnerable to experiences of discrimination. Gendered racial discrimination warrants further study in understanding girls' disruptive behavior and other mental and behavioral health outcomes.

\section{P-18: Female Mice Are More Susceptible to Influenza A Virus Infection and Demonstrate Increased Inflammation and Lung Elastance}

E. Scott Halstead (presenting author), Todd M. Umstead, Michael L. Davies, Linlin Yang, Sanmei Hu, and Zissis C. Chroneos

Department of Pediatrics, Hershey Pulmonary Immunology and Physiology Core; and Department of Microbiology and Immunology, College of Medicine, Pennsylvania State University

Background and Objective: During the $2009 \mathrm{H} 1 \mathrm{~N} 1$ influenza A virus (IAV) pandemic, female sex and pregnancy were risk factors for mortality. Similar sex differences in IAV pathogenicity have been observed in female mice, although the underlying mechanisms have not been well characterized. In this study we sought to measure sex differences in (1) susceptibility to influenza virus; (2) lung inflammation via cytokine and FACS analysis; and (3) lung mechanics at baseline and after influenza virus infection.

Methods: C57BL/6 background wild-type equivalent mice were infected intranasally with the A/8/Puerto Rico/34 influenza strain. Using 14-parameter flow cytometry, the cellular immune response and cytokines/chemokines were measured in bronchoalveolar lavage fluid via Luminex.

Results: Female mice were two-fold more susceptible to IAV, with a lethal dose 50\% (LD50) of 548 fluorescent focus units (FFU) compared with 1,212 FFU for male mice. During IAV infection, female mice demonstrated elevated levels of IL-2, IL18, IP-10, TNF-alpha, and GM-CSF in the airways, corresponding with the presence of more inflammatory monocytes. At baseline, female lungs demonstrated higher elastance, i.e., stiffer lungs $\left(19.5 \pm 0.88\right.$ vs. $\left.15.9 \pm 0.33 \mathrm{~cm} \mathrm{H}_{2} \mathrm{O} / \mathrm{mL}, * P<.02\right)$, and while both sexes demonstrated an increase in lung elastance with IAV infection, the increase was more dramatic in female mice $(67 \%)$ than in males $(43 \%)$.

Conclusions: During IAV infection, female mice demonstrated increased inflammation that correlated with increased lung tissue elastance. Future studies will investigate the role of 
sex hormone-related nuclear factors in IAV inflammation, which may inform future clinical diagnostic and therapeutic studies.

\section{P-19: The Disinhibition Factor: Sexual Well-Being Outcomes Among New IUD and Implant Users After 1 and 3 Months}

Jenny A. Higgins (presenting author), ${ }^{1}$ Jessica N. Sanders, ${ }^{3}$ Mari Palta, ${ }^{2}$ and David K. Turok ${ }^{3}$

${ }^{1}$ Department of Gender and Women's Studies and ${ }^{2}$ Department of Population Health, University of Wisconsin; ${ }^{3}$ Department of Obstetrics and Gynecology, University of Utah

Background and Objective: Although contraceptives are designed for sexual activity, little research documents contraceptives' sexual acceptability. This gap is especially notable for intrauterine devices (IUDs) and implants, the most effective but most underutilized methods available. We sought to document how IUDs and implants affect women's sexual well-being among new-start contraceptive users.

Methods: A baseline survey collected sociodemographic and health information, contraceptive selection criteria, and sexual function measures-including the Female Sexual Function Index (FSFI) and the New Sexual Satisfaction Scale (NSSS). After 1 and 3 months, follow-up surveys assessed FSFI, NSSS, and perceived impact of the method used on sexuality (positive, negative, or no change). We assessed differences from baseline and trends over time by conditional logistic regression.

Results: Among 159 new-start contraceptive users, 20\% selected a copper IUD, $46 \%$ the levonorgestrel IUD, and $34 \%$ the contraceptive implant. Most women said it was extremely important that contraception doesn't reduce libido (77\%) and doesn't interrupt sex (73\%). FSFI and NSSS scores did not change significantly over time. However, across all three methods, participants were significantly more likely to report subjective improvements in their sexual lives $\left(\chi^{2} P<.0001\right)$. At 1 month, $38 \%$ of women reported improvement in their sex life from enrollment, compared to $15 \%$ reporting sexual detractions. By 3 months, $40 \%$ of women reported improvements. Sexual improvements were associated with increased sexual disinhibition. Detractions were largely accounted for by increased vaginal bleeding.

Conclusions: For new IUD and implant users, perceptions of their method's sexual impact may be more important than conventional measures of sexual functioning. While increases in bleeding were associated with women's sexual dislike of their method, IUDs and implants show potential for improving sexuality by enhancing women's security against unintended pregnancy.

\section{P-20: Concordance Between Parental and Provider Reports of HPV Vaccination Among 13- to 17- Year-Olds Participating in NIS-Teen, 2008-2013}

Jacqueline M. Hirth (presenting author), ${ }^{1}$ Yong-Fang Kuo, ${ }^{2}$ Tabassum H. Laz, ${ }^{5}$ Richard E. Rupp, ${ }^{3}$ Jonathan M. Starkey, Mahbubur Rahman, ${ }^{6}$ and Abbey B. Berenson ${ }^{1}$

${ }^{1}$ Center for Interdisciplinary Research in Women's Health, Department of Obstetrics and Gynecology, ${ }^{2}$ Division of Epidemiology and Biostatistics, Preventive Medicine and Community Health, ${ }^{3}$ Department of Pediatrics, and ${ }^{4}$ Institute for Translational Sciences, Medical Branch, University of
Texas; ${ }^{5}$ Health Research and Consulting Services, Dhaka, Bangladesh; ${ }^{6}$ Center for Clinical Epidemiology, St. Luke's International University, Tokyo, Japan

Background and Objective: Parental reports of human papillomavirus (HPV) vaccination are often used to determine adolescents' vaccination status, but the accuracy of parental reports may have changed since the vaccine was introduced. The purpose of this study was to examine concordance of the parent's and provider's report of HPV vaccination.

Methods: The NIS-Teen was used to examine the accuracy of parental reports for HPV vaccination among female teenagers aged 13-17 years between 2008 and 2013. Parental reports were compared with the provider's report. We also examined the proportion of parents that overreported and underreported HPV vaccination in their teenaged children. Multivariable logistic regression models examined associations between adolescent characteristics and concordance of the parent's and provider's report of HPV vaccination.

Results: Overall, there was $84 \%$ concordance between the parent's and provider's report. Concordance varied, however, by race/ethnicity, geographic region, year of the survey interview, and income. Variations in agreement were mainly due to differences in underreporting. Parents of Hispanic (adjusted odds ratio $[\mathrm{aOR}]=.66 ; 95 \%$ confidence interval $[\mathrm{CI}], .57, .75)$ and black teens $(\mathrm{aOR}=.66 ; 95 \% \mathrm{CI}, .58, .76)$ were less likely to correctly report their vaccination status than were parents of white teens. Parents living in the West $(\mathrm{aOR}=.73 ; 95 \% \mathrm{CI}, .65$, .94) were less likely to correctly report vaccination status, compared with those living in the Northeast, and lower income was associated with lower odds of agreement with the provider's report. A later interview year was also associated with a decrease in correct recall of HPV vaccination.

Conclusions: Correct parental report of HPV vaccination in this study was moderate, with much of the disagreement coming from underreporting. These findings indicate that studies that use reports by parents of the vaccination of their teenagers may not be accurate, and that accuracy varies according to sociodemographic variables.

\section{P-21: A Multiple-Goal Lifestyle Intervention Prevents Excessive Gestational Weight Gain in High-Risk Women}

Holly R. Hull, Jeannine Goetz, Debra Sullivan, and Susan Carlson

\section{Medical Center, University of Kansas}

Background and Objective: Overall, 55\% of women gain excessive gestational weight. There has been limited success reported in published interventions to prevent excessive gestational weight gain (GWG). This study examined the effectiveness of a multiple-goal (MG) lifestyle intervention to prevent excessive GWG.

Methods: Pregnant women were recruited at 14 to 18 weeks, and the intervention started at 18 weeks. The intervention included 18 weekly 60-minute lessons led by a registered dietitian. Program evaluation was completed using structured interviews in the MG group.

Results: Fourteen women were enrolled into the MG group, and 12 women were enrolled into the usual care (UC) group. No women were lost from the MG group, and 3 were lost from the UC group. The mean pre-pregnancy BMI $\left(\mathrm{kg} / \mathrm{m}^{2}\right)$ was 29.3 in 
the UC group and 28.4 in the MG group. In the MG group, $29 \%$ of the women gained excessively, while $78 \%$ of the UC group did so $(P<.05)$. Additionally, the UC group gained more weight $(18.4 \mathrm{~kg}$ vs. $11.8 \mathrm{~kg} ; P=.010)$. When the MG group $(\mathrm{n}=9)$ was asked, "What changes did you make due to the intervention?" 33\% responded, "consumed more fruits/vegetables" and "controlled portions," while $22 \%$ said "consumed less sugar." When asked what they liked about the program, 44\% replied "monitoring body weight." When asked what they would recommend for future interventions, responses included "keep a variety of topics" and "set one goal for the entire program."

Conclusions: Women participating in the MG group had lower rates of excessive GWG and lower overall GWG.

\section{P-22: Chronic Type 2 Inflammation Promotes a Unique B Cell Activation Environment}

Kathryn Hulse (presenting author), ${ }^{1}$ Scott Feldman, ${ }^{1}$ Jason Chen, ${ }^{1}$ Richard Kasjanski, ${ }^{1}$ Atsushi Kato, ${ }^{1}$ Julie Poposki, ${ }^{1}$ James Norton, ${ }^{1}$ Lydia Suh, ${ }^{1}$ Roderick G. Carter, ${ }^{1}$

Robert C. Kern, ${ }^{2}$ Stephanie Shintani-Smith, ${ }^{2}$ David B. Conley, ${ }^{2}$ Anju T. Peters, ${ }_{1}^{1}$ Leslie C. Grammer III, ${ }^{1}$ Kathleen E. Harris, ${ }^{1}$ Bruce Tan, ${ }^{2}$ Margrit Urbanek, ${ }^{3}$ and Robert Schleimer ${ }^{1,2}$

${ }^{1}$ Division of Allergy-Immunology, ${ }^{2}$ Department of Otolaryngology - Head and Neck Surgery, and ${ }^{3}$ Division of Endocrinology, Metabolism and Molecular Medicine, Feinberg School of Medicine, Northwestern University

Background and Objective: Chronic rhinosinusitis with nasal polyps (CRSwNP) is characterized by type 2 inflammation and elevated B cells, antibodies, and expression of the extrafollicular B cell marker Epstein-Barr virus-induced protein 2 (EBI2). Although women seem to have more severe disease than men, the mechanisms that drive CRSwNP are not clear. We sought to determine how B cells in nasal polyps become activated.

Methods: Cells were isolated from nasal polyps or tonsil tissue, and B cell phenotype was assessed by flow cytometry. The frequency of antibody-secreting cells was measured by ELISpot assay, and qRT-PRR was used to determine gene expression.

Results: Compared with tonsils, polyps contained elevated levels of plasmablasts $(\mathrm{PB})$ and $\mathrm{EBI} 2+\mathrm{PB}(P<.001$ and $P<.01$, respectively), but fewer naïve and germinal center $B$ cells $(P<.001)$. Expression of germline transcripts, a marker of local $\mathrm{B}$ cell activation, was equivalent in polyps and tonsils, suggesting high levels of local B cell activation in polyps. In vitro, polyps produced significantly higher levels of antibodies compared with tonsils $(P<.01)$, and polyps contained a higher frequency of antibody-secreting cells $(P<.05)$. Interestingly, coculture of $B$ cells with group 2 innate lymphoid cells (ILC2s) increased the frequency of EBI2+ B cells $(P<.05)$.

Conclusions: These findings indicate that $\mathrm{B}$ cell activation in polyps is distinct from the classical activation that occurs in tonsils. Further, they suggest that type 2 inflammation, and in particular, ILC2s, may play an important role in activation of B cells during chronic airway inflammation.

\section{P-23: Public Health Messages About Perinatal Marijuana Use in an Evolving Policy Context}

Marian Jarlenski (presenting author), ${ }^{1}$ Jennifer Zank, ${ }^{1,2}$ Jill Tarr, ${ }^{3}$ and Judy Chang ${ }^{3,4}$
${ }^{1}$ Department of Health Policy and Management, Graduate School of Public Health, ${ }^{2}$ Children's Hospital of Pittsburgh, ${ }^{3}$ Magee-Womens Research Institute, and ${ }^{4}$ Department of Obstetrics, Gynecology and Reproductive Sciences and Department of Internal Medicine, University of Pittsburgh

Background and Objective: Marijuana is the most commonly used substance in pregnancy in the U.S., and in utero exposure poses health risks for infants. More than one-third of U.S. births occur in states where marijuana use is legal for medical or recreational purposes. The objective of this study was to analyze the volume and content of health communications published by state and federal public health agencies about perinatal marijuana use.

Methods: In early 2016, we systematically searched 51 state and 4 federal public health agencies' websites for information about perinatal marijuana use. Two investigators independently coded the published public health content using a 12-item instrument $(\kappa$ range $=.58-1.00)$.

Results: On the 55 public health websites searched, 1 federal agency and 10 state agencies had published information about perinatal marijuana use. Most content was targeted to the public, although 3 agencies had content targeted to health care providers. Among public health agencies with published content about perinatal marijuana use, 8 mentioned health effects of marijuana during breastfeeding, 5 included resources to stop using marijuana, 5 mentioned implications of marijuana use for infant safety and parenting, and 2 mentioned potential legal consequences. Common messages about perinatal health effects included pregnancy complications and longer-term cognitive effects in infants and children exposed in utero. The most common message for quitting perinatal use was to talk to a physician.

Conclusions: Despite rapidly changing state policies regarding the legal status of marijuana use, the volume of public health messages about perinatal marijuana use is low, and the content of messages differs across state agencies.

\section{P-24: Developmental Programming of Pulmonary Vascular Dysfunction by Perinatal Hypoxia Is Sex Dependent}

Colleen Glyde Julian (presenting author), Gabriel Wolfson, and David A. Schwartz

\section{Department of Medicine, School of Medicine, University of Colorado}

Background and Objective: Perinatal exposures alter susceptibility to adult-onset disease. Because developmental processes that are required for efficient pulmonary gas transfer are incomplete at birth, the lung and its pulmonary circulation are particularly vulnerable. Our human studies demonstrate that perinatal hypoxia $\left(\mathrm{P}_{\mathrm{HX}}\right)$ raises the risk of pulmonary vascular dysfunction (PVD) among male high-altitude residents. We sought to develop a murine model in which the degree and timing of environmental hypoxia could be strictly controlled to directly test the hypothesis that $\mathrm{P}_{\mathrm{HX}}$ increases susceptibility to hypoxia-induced PVD during adulthood.

Methods: Experiments were performed using three groups of C57/BL6 mice distinguished by the timing of normoxic $\left(\mathrm{N}_{\mathrm{X}}\right)$ or hypoxic $\left(\mathrm{H}_{\mathrm{X}}\right)$ exposure: $\mathrm{Nx}$ controls $(\mathrm{C}-\mathrm{C}), \mathrm{P}_{\mathrm{HX}}+$ adult $\mathrm{H}_{\mathrm{X}}$ $\left(\mathrm{P}_{\mathrm{HX}}-\mathrm{H}_{\mathrm{X}}\right)$, and perinatal $\mathrm{N}_{\mathrm{X}}+$ adult $\mathrm{H}_{\mathrm{X}}\left(\mathrm{C}-\mathrm{H}_{\mathrm{X}}\right)$. For the perinatal normoxia and $\mathrm{H}_{\mathrm{X}}\left(\mathrm{PH}_{\mathrm{X}}\right.$ or $\left.\mathrm{H}_{\mathrm{X}}\right)$, pregnant dams were placed in a hyperbaric $(760 \mathrm{mmHg})$ or hypobaric chamber $(375 \mathrm{mmHg})$ from gestational day 14 through postnatal day 4. All animals 
were housed in a normoxic environment from postnatal day 5 to 3 weeks. Adult exposures lasted from 3 to 8 weeks of age. Pulmonary hemodynamic (via echocardiography) and right ventricular systolic pressure measurements were performed in 8 -week-old offspring. Intracardiac blood samples were used to measure hematocrit. ANOVA with multiple comparisons was used to identify differences between groups.

Results: $\mathrm{P}_{\mathrm{HX}}$ reduced pulmonary artery acceleration time and pulmonary valve peak flow velocity and increased right ventricular wall thickness, right ventricular systolic pressure, and hematocrit in male pups only.

Conclusions: Our studies demonstrate that $\mathrm{P}_{\mathrm{HX}}$ increases hypoxia-induced PVD and that such effects are sex dependent.

\section{P-25: The Urinary Microbiome: Does It Play a Role in Urge Urinary Incontinence and Its Severity?}

Lisa Karstens (presenting author), ${ }^{1,2}$ Mark Asquith, ${ }^{3}$ Sean Davin, ${ }^{3}$ Patrick Stauffer, ${ }^{3}$ Damien Fair, ${ }^{4}$ William T. Gregory, ${ }^{2}$ Jim Rosenbaum, ${ }^{3}$ Shannon McWeeney, ${ }^{1}$ and Rahel Nardos ${ }^{2}$

${ }^{1}$ Division of Bioinformatics and Computational Biology,

${ }^{2}$ Division of Urogynecology and Reconstructive Surgery,

${ }^{3}$ Division of Arthritis and Rheumatic Diseases, and ${ }^{4}$ Department of Behavioral Neuroscience, Oregon Health \& Science University

Background and Objective: Traditionally, the urinary tract has been thought to be sterile in the absence of a clinically identifiable infection. However, recent evidence suggests that the urinary tract harbors a variety of bacteria, known collectively as the urinary microbiome, even when clinical cultures are negative. Whether these bacteria promote health or contribute to disorders such as urge urinary incontinence (UUI) remains unknown. The goal of this prospective study was to determine how the urinary microbiome differs between women with and without UUI and to identify if characteristics of the urinary microbiome are associated with UUI severity.

Methods: We collected urine from clinically wellcharacterized women with UUI $(\mathrm{n}=10)$ and normal bladder function $(\mathrm{n}=10)$. To characterize the resident microbial community, we amplified the bacterial 16S rRNA gene by PCR and sequenced it with Illumina MiSeq. Linear models based on the negative binomial distribution were used to identify differentially abundant bacteria, and Pearson's correlation coefficient was used to identify relationships between diversity and symptom severity.

Results: We identified the presence of a polymicrobial community in the female bladder. We found 14 bacteria that have significantly different relative abundances between control and UUI samples. Furthermore, we established that increasing symptom severity is significantly associated with decreased microbial diversity in women with UUI.

Conclusions: Our study provides evidence that supports the existence of the urinary microbiome. Our results suggest that the urinary microbiome may play an important role in the pathophysiology of UUI and that loss of diversity may be associated with clinical severity.

\section{P-26: Association of Serum Adiponectin and Adipsin with Systemic Sclerosis and Its Subtypes}

Benjamin Korman (presenting author), ${ }^{1}$ Roberta Marangoni, ${ }^{1}$ Monique Hinchcliff, ${ }^{1}$ Sanjiv Shah, ${ }^{2}$ Mary Carns, ${ }^{1}$ Rosalind Ramsey-Goldman, ${ }^{1}$ and John Varga ${ }^{1}$
${ }^{1}$ Division of Rheumatology and ${ }^{2}$ Division of Cardiology, Department of Medicine, Feinberg School of Medicine, Northwestern University

Background and Objective: Systemic sclerosis (SSc) is a multisystem autoimmune fibrotic disorder that disproportionately affects women. Recent evidence suggests that adipose tissue plays a role in the pathogenesis of this disorder. Adipokines are hormones that are secreted by adipose tissue and affect inflammation, vascular function, and fibrosis, the hallmarks of SSc. In this study, we sought to assess whether serum adipokine levels correlate with SSc and relevant disease subtypes.

Methods: Multiplex luminex-based adipokine assays to assess visfatin, resistin, leptin, adipsin, and adiponectin levels were performed in $198 \mathrm{SSc}$ patients and 33 control individuals. All patients underwent standard clinical assessments, including autoantibody testing and determination of the modified Rodnan skin score (MRSS), had pulmonary function tests and highresolution chest $\mathrm{CT}$ scans, and underwent echocardiograms within 6-12 months of serum collection. Echocardiograms were read using a standardized research protocol.

Results: Visfatin, resistin, and leptin levels did not show significant associations with SSc or its subtypes. Adipsin was associated with SSc. Both adipsin and adiponectin showed strong associations with antibody status and the MRSS, although limited cutaneous SSc and diffuse cutaneous SSc showed very different patterns, and associations were not seen within these groups. Adipsin was associated with multiple markers of cardiac involvement in SSc.

Conclusions: This study affirms the association of adiponectin with SSc and finds new associations between adipsin and $\mathrm{SSc}$, antibody status, the MRSS, pulmonary hypertension, and cardiac parameters. Adipokine profiles may be useful in assessing risk and monitoring internal organ manifestations of SSc.

\section{P-27: Sex Differences in Cumulative Exposure to Tenofovir as Determined via Hair Levels in VOICE, iPrEx OLE, and PrEP Demo}

Catherine A. Koss (presenting author), ${ }^{1}$ Peter Bacchetti, ${ }^{2}$ Edward Livant, ${ }^{4}$ Howard Horng, ${ }^{3}$ Nyaradzo Mgodi, ${ }^{5}$ Brenda Gati, ${ }^{6}$ Kailazarid Gomez Feliciano, ${ }^{7}$ Stephanie Horn, ${ }^{7}$ Albert Liu, ${ }^{8}$ David Glidden, ${ }^{2}$ Robert Grant, ${ }^{1}$ Leslie Z. Benet, ${ }^{3}$ Alexander Louie, ${ }^{3}$ Z. Mike Chirenje, ${ }^{5}$ Jeanne Marrazzo, ${ }^{9}$ and Monica Gandhi, ${ }^{1}$ on behalf of the MTN-003 Protocol Team

${ }^{1}$ Department of Medicine, ${ }^{2}$ Department of Epidemiology and Biostatistics, and ${ }^{3}$ School of Pharmacy, University of California, San Francisco; ${ }^{4}$ University of Pittsburgh; ${ }^{5}$ University of Zimbabwe-University of California,

San Francisco Collaborative Research Program, Harare, Zimbabwe; ${ }^{6}$ Makerere University-Johns Hopkins University Research Collaboration, Kampala, Uganda; ${ }^{7}$ FHI 360, Durham, North Carolina; ${ }^{8}$ San Francisco Department of Public Health; ${ }^{9}$ Department of Medicine, University of Alabama, Birmingham

Background and Objective: Pre-exposure prophylaxis (PrEP) protected individuals at risk for HIV but uninfected from acquiring HIV in several trials, and is broadly recommended. In the VOICE trial, however, tenofovir disoproxil fumarate (TDF)/ emtricitabine (FTC)-based PrEP did not prevent HIV acquisition in women. Tenofovir (TFV) was detected in plasma, reflecting exposure/adherence in recent days, in fewer than one-third of 
participants. Hair concentrations represent cumulative drug exposure/adherence over weeks to months. The objective of this study was to compare hair concentrations of TFV from participants in the VOICE trial and 2 open-label PrEP studies in order to evaluate sex differences in cumulative exposure and adherence to PrEP.

Methods: Hair samples were collected from a subset of VOICE participants who had been assigned to the TDF/FTC group. TFV hair concentrations, measured via liquid chromatography tandem mass spectrometry, were compared with selfreported adherence and with hair levels in 2 open-label PrEP studies in men and transwomen. The median age of the 47 VOICE participants was 28.4 years, $56.3 \%$ were married, and their median time on the study before hair collection was 1.1 years.

Results: There was little correlation between self-reported adherence and hair concentrations. TFV hair levels were undetectable in $45 \%$ of the 47 women in VOICE vs. in $25 \%$ of 838 person-visits in the iPrEx OLE study $(P=.006)$ and in $2 \%$ of 875 person-visits in PrEP Demo $(P<.001)$. Median TFV hair concentrations (in $\mathrm{pg} / \mathrm{mg}$ ) were 2.4 (IQR BLQ-16.8) in VOICE, $17.1(2.2-35.2)$ in iPrEx OLE $(P<.001)$, and $34.5(26.1-44.4)$ in PrEP Demo $(P<.001)$, corresponding to an estimated 0.2, 2.9, and 6.0 TDF doses/week taken, respectively.

Conclusions: This VOICE hair substudy is the first to examine cumulative TFV exposure/adherence via hair concentrations in women, among whom the failure to detect the drug was far higher than it was in men in open-label PrEP studies. Further examination of behavioral and biological factors leading to sex differences in drug concentrations is needed to optimize this important prevention modality in women.

\section{P-28: Reproductive Coercion, Intimate Partner Violence, and Pregnancy Risk Among Adolescent Women in Foster Care}

Heather L. McCauley (presenting author) ${ }^{1}$ and Elizabeth Miller ${ }^{2}$

${ }^{1}$ Department of Pediatrics, School of Medicine; and ${ }^{2}$ Division of Adolescent and Young Adult Medicine, Children's Hospital of Pittsburgh, Medical Center, University of Pittsburgh

Background and Objective: Reproductive coercion (RC) is an increasingly recognized mechanism linking intimate partner violence (IPV) and poor reproductive health among young women, but studies on RC have focused on clinical or college samples. The objective of this study was to document the prevalence of RC in a community-based sample and determine whether RC was associated with pregnancy risk.

Methods: Adolescent women aged 16-24 years were recruited from youth-serving agencies affiliated with the Allegheny County, Pennsylvania, foster care system $(n=121)$ to complete a computer-based survey. Associations between RC and study outcomes were assessed via descriptive statistics and logistic regression.

Results: Lifetime RC was reported by $34 \%$ of the sample, while $63 \%$ of women had experienced IPV. RC was associated with elevated odds of IPV (adjusted odds ratio $[\mathrm{AOR}]=4.1,95 \%$ confidence interval $[\mathrm{CI}], 1.5,11.1)$, gang rape $(\mathrm{AOR}=3.2,95 \%$ $\mathrm{CI}, 1.02,10.0$ ), having a sex partner 5 or more years older (AOR $=12.0,95 \% \mathrm{CI}, 4.0,36.7)$, using alcohol or drugs before sex $(\mathrm{AOR}=4.7,95 \% \mathrm{CI}, 1.9,11.5)$, and trading sex for money or drugs $(\mathrm{AOR}=6.0,95 \% \mathrm{CI}, 1.6,22.2)$, in models controlling for demographics. Two-fifths and one-quarter of women reported pregnancy and unwanted pregnancy, respectively. In adjusted models, RC increased women's odds for pregnancy outcomes more than two-fold (pregnancy, $\mathrm{AOR}=2.4,95 \% \mathrm{CI}, 1.03,5.7$; unwanted pregnancy, AOR $=2.7,95 \% \mathrm{CI}, 1.1,6.7$ ).

Conclusions: Practitioners working with pregnant or parenting foster youth may benefit from integrating targeted discussion about RC into their assessments and ensuring that their clients have access to confidential reproductive and sexual health services.

\section{P-29: Sex Differences in Medication Use Following a Dementia Diagnosis in Older Adults}

Daniela C. Moga (presenting author), ${ }^{1,2}$ Qishan $\mathrm{Wu},{ }^{2}$ and Gregory Jicha ${ }^{3,4}$

${ }^{1}$ Department of Pharmacy Practice and Science, ${ }^{2}$ Department of Epidemiology, ${ }^{3}$ Department of Neurology, and ${ }^{4}$ Sanders-Brown Center on Aging, University of Kentucky

Background and Objective: Alzheimer's disease and other dementias represent an important and growing public health issue. Early diagnosis is often elusive, and misdiagnosis can lead to inappropriate treatment. Previous research has shown an association between misdiagnosis and inappropriate treatment, as well as between sex and a misdiagnosis of dementia. Our study examined sex differences related to treatment following a diagnosis of dementia, specifically whether women and men were treated to similar extents with cognitive enhancers and potentially inappropriate medications (i.e., psychotropic medications).

Methods: We identified participants aged 65 years or older enrolled in the National Alzheimer's Coordinating Center (NACC) and newly diagnosed with dementia between 2005 and 2014. We evaluated sex differences in clinical diagnosis and investigated differences in the use of cognitive enhancers and psychotropic medications between women and men following diagnosis while they were participating in the cohort. Multivariable logistic regression was performed to investigate the role of sex and determine the adjusted odds ratios (aORs) with associated $95 \%$ confidence intervals (CIs).

Results: Of the 31,872 NACC enrollees, we identified 2,565 (1,329 women) with incident dementia. Of these, 2,387 (1,239 women) were aged $\geq 65$ years at diagnosis and were included in our analyses. Although more women were diagnosed with Alzheimer's disease (66\% vs. $60 \%$ ), they used cognitive enhancers less ( $45 \%$ vs. $56 \%$; aOR $=0.64,95 \% \mathrm{CI}, .64-.76)$ and psychotropic medication more $(47 \%$ vs. $36 \%$; aOR $=1.62,95 \% \mathrm{CI}$, 1.33-1.98) than men.

Conclusions: Our study showed that women are treated less with cognitive enhancers and more with potentially inappropriate medications (i.e., psychotropic medications); future studies are needed to investigate the optimal approach to address these disparities.

\section{P-30: It Weigh(t)s on You: Everyday Discrimination and Obesity Among Latino Women and Men}

Kristine M. Molina (presenting author) ${ }^{1}$

Noemi Rivera-Olmedo, ${ }^{1}$ and Milagros C. Rosal $^{2}$

${ }^{1}$ Department of Psychology, University of Illinois at Chicago; ${ }^{2}$ Division of Preventive and Behavioral Medicine, Department of Medicine, Medical School, University of Massachusetts 
Background and Objective: Discrimination is robustly associated with depression, and depression is linked to obesity. These are 2 health indicators in which disparities exist by gender and ethnicity. However, no study to date has examined whether discrimination is indirectly associated with accumulation of excess body fat via increased depressive symptoms, and whether these effects differ by gender. We examined (1) the indirect effects of everyday discrimination on body mass index (BMI) and waist circumference, and (2) whether this effect differed between Latino women and men.

Methods: Participants included 602 adults of predominantly Latino Caribbean heritage recruited from a community health center in Lawrence, Massachusetts. We used the Everyday Discrimination Scale and the Center for Epidemiological Studies Depression Scale. Anthropometric measures included BMI (weight [pounds]/height $[\text { inches }]^{2} \times 703$ ) and waist circumference (measured twice in centimeters; the average was used).

Results: Descriptive analyses showed that women reported significantly higher levels of depressive symptoms and genderspecific BMI levels, whereas men reported higher levels of discrimination. Structural equation modeling analyses revealed, however, that the hypothesized model fit the data adequately for both men and women, with no significant gender differences. Thus, results for the total sample showed that discrimination was directly associated with increased depressive symptoms, higher levels of BMI, and larger waist circumference.

Conclusions: Rather than an indirect effect, our research found support for a direct, positive association between discrimination and obesity indicators, suggesting that discrimination may be implicated in obesity disparities for both Latino women and men. Future studies should examine potential gender-specific mechanisms by which discrimination may impact obesity.

\section{P-31: Awareness of and Reactions to the Controversy Over Mammography Among Ethnic Immigrant Women}

Rebekah H. Nagler, Jennifer A. Lueck, and Lauren S. Gray

School of Journalism and Mass Communication,

University of Minnesota

Background and Objective: The past 20 years have witnessed substantial expert disagreement about the use of mammography to screen for breast cancer, and this disagreement routinely plays out in the media. Evidence suggests that some women are aware of the controversy over mammography, but less is known about whether ethnic immigrant and other underserved women have heard about this controversy and, if so, how they react to it. The objective of this study was to explore awareness among ethnic immigrant women of the mammography controversy and their reactions to it.

Methods: We conducted 6 community-engaged focus groups $(n=53)$ with women aged 35-55 years from 3 ethnic immigrant communities (Somali, Latina, and Hmong). A grounded theory approach was used to identify themes; NVivo 10 was used to enhance analyses.

Results: Several themes emerged: 1) low awareness of the mammography controversy across groups, despite self-reported attention to health information; 2) high intentions to be screened, even after being told about the controversy; 3) few reported discussions with clinicians of mammography's risks and benefits; 4) substantial interest in learning more about mammography and breast cancer, but some low self-efficacy to obtain such information; and 5) questions about whether health recommendations matter and what qualifies as evidence.

Conclusions: Given ongoing expert disagreement about mammography screening, it is important for clinicians to help women understand mammography's risks and benefits so that they can make an informed choice. This is particularly critical for ethnic immigrant and other underserved women, who may be less able to access, attend to, process, retain, and act on health information (a phenomenon known as communication inequality).

\section{P-32: Iterative Testing of a Mobile Health Program for Postpartum Women at Elevated Risk for Cardiometabolic Disease}

Jacinda M. Nicklas (presenting author), ${ }^{1}$ Jennifer A. Leiferman, ${ }^{3}$ Sheana S. Bull, ${ }^{3}$ Cynthia N. Hazel, ${ }^{3}$ Susan J. Gilbert, ${ }^{4}$ Dean A. Hovey, ${ }^{4}$ and Linda A. Barbour ${ }^{2}$

${ }^{1}$ Division of General Internal Medicine and ${ }^{2}$ Division of Endocrinology, Metabolism, and Diabetes, School of Medicine, University of Colorado; ${ }^{3}$ Department of Community and Behavioral Health, Colorado School of Public Health; ${ }^{4}$ Digifit, Inc.

Background and Objective: Postpartum women whose conditions during pregnancy indicate elevated risk for cardiovascular disease and diabetes can decrease their risk via postpartum weight loss, and yet traditional face-to-face interventions are often unsuccessful. Our objective was to iteratively test and improve a theory-based mobile health (mHealth) program designed to help overweight/obese postpartum women decrease their risk for cardiometabolic disease.

Methods: The mHealth Fit After Baby program delivers daily evidence-based content; facilitates tracking of weight, diet, and steps; and provides weekly coaching. We conducted 3 rounds of beta-testing focused on usability, navigability, and acceptability. We analyzed feedback and implemented changes to the app and program after each round of testing.

Results: Twenty-six postpartum women participated in pilot testing (4 in round 1,13 in round 2 , and 9 in round 3 ). Their mean age was $33(\mathrm{SD}=4)$ years; and mean BMI (weight [kilograms]/ height $[\text { meters }]^{2}$ was $33(\mathrm{SD}=6) ; 92 \%$ were White and $8 \%$ were Asian. Participants' most recent pregnancy was complicated by preeclampsia $(35 \%)$, preterm delivery $(31 \%)$, gestational hypertension (42\%), gestational diabetes (27\%), and/or delivery when the newborn was small for gestational age $(15 \%)$. We demonstrated an increase in participant satisfaction and in the number of coaching interactions from round 1 to round 3. The primary themes from feedback were to 1) improve navigation through a different home screen; 2) increase content focused on challenges faced by new mothers and on diet (including recipes) and exercise (including workouts); and 3) better describe points and badges and how to earn them.

Conclusions: Through a process of iterative testing we were able to improve the mHealth Fit After Baby program in preparation for a larger randomized trial.

\section{P-33: Milk Production in Mothers With and Without Signs of Insulin Resistance}

Laurie Nommsen-Rivers (presenting author), ${ }^{1}$ Sarah Riddle, ${ }^{1}$ Amy Thompson, ${ }^{2}$ Laura Ward, ${ }^{1}$ and Erin Wagner ${ }^{1}$ 
${ }^{1}$ Cincinnati Children's Hospital Medical Center and ${ }^{2}$ College of Medicine, University of Cincinnati

Background and Objective: Maternal obesity is associated with a shortened duration of breastfeeding. Insulin resistance (IR) is a physiologic hallmark of obesity and may underlie lactation difficulties in obese and other insulin-resistant mothers. Our objective was to compare 24-hour milk production in mothers with signs of IR versus mothers without such signs.

Methods: We examined baseline data from the MALMS study, a randomized placebo-controlled trial using metformin to augment low milk supply. Baseline eligibility included milk production insufficient to sustain healthy newborn weight gain, mother 1-8 weeks postpartum, healthy term infant, and desire to increase milk production. At baseline, all women recorded milk production over 24 hours and underwent evaluation for trial eligibility, including the following signs of IR: fasting plasma glucose $\geq 95 \mathrm{mg} / \mathrm{dL}$, abdominal obesity, recent gestational diabetes, or polycystic ovary syndrome. We compared baseline 24hour milk production in IR versus no-IR groups with the $t$-test.

Results: Of 40 women with baseline measurements, 27 (68\%) exhibited signs of IR. Mean ages $( \pm$ SD) of the mother $(31 \pm 5$ years) and infant ( $4 \pm 2$ weeks) were similar between IR and noIR groups, but milk production was significantly lower in IR versus no IR: $259 \pm 199$ vs. $465 \pm 191 \mathrm{~mL} /$ day, respectively, $P=.004$.

Conclusions: Exclusively breastfeeding infants typically consume $550-850 \mathrm{~mL}$ of milk per day. While poor lactation management may have contributed to low production in some of our participants, our results strongly suggest an additional deficit attributable to IR. Metformin is a first-line antidiabetic drug, but our trial is the first to determine its feasibility in treating low milk production.

Funding source: NIH 5 K12 HD051953 (PI, Joel Tsevat), Bridging Interdisciplinary Research Careers in Women's Health (BIRCWH award to L N-R).

\section{P-34: Induction of Cancer Stem Cell Markers Following Treatment with Molecularly Targeted Therapies in Epithelial Ovarian Cancer Cell Lines}

Harsh B. Pathak (presenting author), Jeff Hirst, and Andrew K. Godwin

Department of Pathology and Laboratory Medicine, Medical Center, University of Kansas

Background and Objective: New treatment options that improve survival of patients with epithelial ovarian cancer (EOC) are sorely needed. Major hurdles include development of drug resistance, leading to tumor recurrence. Recent studies suggest that short-term treatment with frontline therapy drugs results in enrichment of tumor cells with cancer stem cell-like properties. These stem-like tumor cells are thought to promote eventual disease recurrence and death in these women. A variety of molecularly targeted drugs have been evaluated in EOC patients and either shown no efficacy or an initial response but no improvement in overall survival. We sought to evaluate whether targeted therapies that have been used in phase 2 clinical trials of EOC patients promote stem cell-like phenotypes in EOC cells.

Methods: TaqMan assays were used to measure gene expression following drug treatment of the EOC cell line A1847.

Results: Following treatment with high concentrations of targeted therapies, drug-resistant tumor cells that survived ex- pressed significantly elevated levels of stem cell markers, including ALDH1, KLF4, NANOG, OCT4, and SOX2. The degree of expression varied by drug type. In many instances, the residual cells were capable of repopulating the cultures after the drug was removed.

Conclusions: EOC is a heterogeneous disease with subpopulations of tumor cells having stem cell-like properties. Drugs targeting activated signaling pathways in EOC will not be able to cure this disease when used as single agents. Combining these types of drugs with drugs that specifically target stem cell pathways may provide more efficacious outcomes in EOC patients.

\section{P-35: Exploring Provider Decisions to Withdraw Life-Sustaining Therapy After Cardiac Arrest via Simulated Vignettes Varying by Race and Gender}

Sarah M. Perman (presenting author), ${ }^{1}$ Allan V. Prochazka, ${ }^{2}$ Edward P. Havranek, ${ }^{3}$ and Stacie L. Daugherty ${ }^{3}$

${ }^{1}$ Department of Emergency Medicine, ${ }^{2}$ Department of Medicine, and ${ }^{3}$ Division of Cardiology, School of Medicine, University of Colorado

Background and Objective: Despite worse cardiac arrest characteristics than men, women have better survival to hospital admission, although lower survival to hospital discharge. We sought to explore the role of race and gender in providers' decisions to withdraw life-sustaining therapy (LST) in comatose survivors of cardiac arrest.

Methods: Faculty and trainees in cardiology, emergency medicine, neurology, and critical care were sent 2 clinical vignettes that varied by race/gender. Each vignette described a comatose cardiac arrest patient with similar neuroprognostic predictors who was treated with therapeutic hypothermia. Participants were asked if at 36 hours post-arrest they would recommend continued LST versus withdrawing LST, and what was the likelihood of (1) good neurologic recovery, and (2) survival to hospital discharge.

Results: Of 357 individuals contacted, 171 (48\%) responded. Recommendations for withdrawing LST did not differ by race (Caucasian $17.8 \%$ vs. African American 18.6\%) or gender (male $16.9 \%$ vs. female $19.5 \%$ ). Participants gave similarly dismal neurologic prognoses and prospects for survival by gender as well as for Caucasians versus African Americans. Trainees were more likely to recommend withdrawing LST than were attending physicians (22\% vs. $14 \%$ ). When queried on the appropriate timing for neuroprognostication, $12 \%$ of respondents reported that no such guideline exists, and 29\% felt that neuroprognostication at $\leq 48$ hours is appropriate.

Conclusions: In simulated patient scenarios, race and gender did not result in varied recommendations. The guideline for delaying neuroprognostication until 72 hours was not always adhered to (18\% did not adhere to it), and projections for recovery were worse than the literature suggests.

\section{P-36: Sexually Dimorphic Increase in Brain Kynurenic Acid After Sleep Deprivation}

Ana Pocivavsek (presenting author), Annalisa M. Baratta, Austin D. Buchla, and Marian A.R. Thomas

Maryland Psychiatric Research Center, Department of Psychiatry, School of Medicine, University of Maryland 
Background and Objective: Restful periods at night have been associated with memory consolidation, whereas prolonged periods of poor sleep quality result in neurocognitive dysfunction and memory impairment. Our current experiments were designed to test the hypothesis that kynurenic acid (KYNA), a neuroactive metabolite of the kynurenine pathway (KP) of tryptophan degradation implicated in disrupting memory consolidation, is elevated in the brain after disturbances in sleep.

Methods: As sleep patterns are sexually dimorphic and fluctuations in ovarian hormones negatively affect sleep, we investigated the effect of sleep deprivation (SD) on KP metabolism in both male and female rodents. Adult rats were sleep deprived by gentle handling for $6 \mathrm{~h}$, and KP metabolites were analyzed in the brain and plasma immediately after SD.

Results: In the hippocampus, KYNA levels were elevated by $296 \%$ in male rats (control: $74 \pm 10$ fmoles $/ \mathrm{mg}$ protein; SD: $293 \pm 77$ fmoles/mg protein). In striking contrast, hippocampal KYNA levels in female animals were not significantly altered after SD (control: $38 \pm 3$ fmoles/mg protein; SD: $48 \pm 6$ fmoles/ $\mathrm{mg}$ protein). In the serum, no significant changes in KYNA or its bioprecursor kynurenine were observed in male or female rats.

Conclusions: These data demonstrate a striking sexual dimorphism in the elevation of brain KYNA after an acute period of SD. Ongoing experiments in our laboratories are designed to examine the functional implications of this phenomenon and to assess the possible role of estrogen and other hormones in protecting the female rodent from an increase in brain KYNA levels after SD.

\section{P-37: Associations of Peripheral Endothelial Function and Arterial Stiffness with Measurements of Glucose Tolerance and Metabolic Dysfunction in Obese Women with Polycystic Ovary Syndrome}

Sushant Ranadive (presenting author), ${ }^{1}$ Antigoni Z. Lalia, ${ }^{2}$ Michael J. Joyner, ${ }^{1,3}$ Amir Lerman, ${ }^{4}$ and Alice Y. Chang ${ }^{2}$

${ }^{1}$ Department of Anesthesiology; ${ }^{2}$ Division of Endocrinology, Diabetes, and Nutrition, Department of Internal Medicine;

${ }^{3}$ Department of Physiology and Biomedical Engineering; and

${ }^{4}$ Department of Cardiology, College of Medicine, Mayo Clinic

Background and Objective: Polycystic ovary syndrome (PCOS) is a common disorder of androgen excess that is strongly associated with insulin resistance, obesity, endothelial dysfunction, and arterial stiffness. In lean women with PCOS without insulin resistance, there is greater peripheral endothelial dysfunction without differences in arterial stiffness compared with matched controls. We sought to determine in obese women with PCOS whether characteristics associated with obesity and insulin resistance correlated differently with peripheral endothelial function than with measurements of arterial stiffness.

Methods: Glucose was measured during a 3-hour oral 75gram oral glucose tolerance test (OGTT) in 18 obese women (32.9 \pm 6.2 years) diagnosed with PCOS with androgen excess and anovulation. Peripheral flow-mediated dilation was assessed using digital arterial tonometry and calculated as the reactive hyperemia index (RHI). Pulse wave velocity (PWV) and augmentation index (AIx) were obtained using applanation tonometry. Associations of clinical variables with RHI, PWV, and AIx were evaluated with linear regression analysis.

Results: Nearly two-fifths (38.9\%) of participants had an RHI $<2$ and higher glucose during the OGTT than women with an RHI $>2$. RHI did not correlate with dyslipidemia but was asso- ciated with weight and waist circumference. PWV and AIx were associated with total cholesterol, HDL, and LDL, but they did not correlate consistently with glucose throughout the OGTT.

Conclusions: In obese women with PCOS, peripheral endothelial function was associated with different measurements of metabolic dysfunction than was arterial stiffness.

Sources of Research Support: Building Interdisciplinary Careers in Women's Health Award K12HD065987 to AYC; CTSA Grant Number UL1 TR000135 from the National Center for Advancing Translational Sciences, St. Jude Medical Foundation, and the Mayo Foundation.

\section{P-38: Poly-L-Lactic Acid Corrosion Casting for Microvascular Scaffold Fabrication: Implications for Breast Reconstruction}

Dino Ravnic (presenting author), ${ }^{1}$ Sulei $\mathrm{Xu},{ }^{2} \mathrm{Xiang} \mathrm{Li},{ }^{2}$ Surge Kalaba, ${ }^{3}$ Ping He, ${ }^{2}$ and Jian Yang ${ }^{3}$

${ }^{1}$ Department of Surgery, ${ }^{2}$ Department of Cellular and Molecular Physiology, and ${ }^{3}$ Department of Cellular and Molecular Physiology, College of Medicine, Pennsylvania State University

Background and Objective: Post-mastectomy breast reconstruction has become the standard of care, with autologous adipocutaneous free tissue transfer gaining popularity despite its potential for surgical morbidity and limited availability. Tissue engineering promises therapeutic potential, but its clinical relevance is hampered by the ability to recreate a perfusable vasculature that nourishes the surrounding parenchyma. Corrosion casting uses an injectable toxic and non-biodegradable resin to precisely cast blood vessels in $3 \mathrm{D}$, and after parenchymal digestion it may prove useful in microvascular scaffold fabrication. We decided to evaluate the potential of an FDA-approved dermal filler (poly-L-lactic acid [PLLA]) to broaden corrosion casting approaches to the engineering of microvascular tissue by pursuing 2 objectives: 1) document its suitability as an intravascular injectable biopolymer, and 2) evaluate its ability to promote endothelial cell adhesion and viability.

Methods: Increasing concentrations of PLLA were dissolved in chloroform. Utilizing a rat liver model $(n=9)$ the hepatic vessels were flushed free of blood, fixed, perfused with PLLA, explanted, and then allowed to harden. Using microscopy, polymer retention and vascular detail were examined. Human umbilical vein endothelial cells were cultured on PLLA that had been coated with either collagen or fibronectin.

Results: PLLA $20 \mathrm{mg} / \mathrm{ml}$ offered perfusability while maintaining vascular detail. Fibronectin- coated PLLA allowed for endothelial cell seeding and viability.

Conclusions: PLLA may serve as an injectable nontoxic biopolymer during corrosion casting, and after parenchymal digestion it may offer a microvascular scaffold that is amenable to endothelial and subsequent adipocutaneous seeding. Timed PLLA degradation allows for luminal creation, and its perfusability would accelerate the expansion of tissue engineering to patients undergoing breast reconstruction.

\section{P-39: Reduced Airway SIgA in Women with COPD Is Associated with Estrogen Receptor Signaling}

Bradley W. Richmond (presenting author), ${ }^{1,2}$ Rui-Hong Du, ${ }^{1}$ Dawn C. Newcomb, ${ }^{1}$ Pierre Massion, ${ }^{1,3,4}$ Timothy S. Blackwell, ${ }^{1-4}$ and Vasiliy V. Polosukhin ${ }^{1}$ 
${ }^{1}$ Department of Medicine, Division of Allergy, Pulmonary, and Critical Care Medicine; ${ }^{2}$ Department of Cell and Developmental Biology; and ${ }^{3}$ Department of Cancer Biology, School of Medicine, Vanderbilt University; ${ }^{4}$ Department of Veterans Affairs Medical Center, Nashville, Tennessee

Background and Objective: Emerging evidence suggests that localized secretory immunoglobulin A (SIgA) deficiency in small airways plays a causal role in the pathogenesis of chronic obstructive pulmonary disease (COPD). Delivery of SIgA to the mucosal surface is dependent upon expression of the polymeric immunoglobulin receptor (pIgR), which binds IgA at the basolateral membrane of airway epithelial cells and facilitates receptor-mediated transcytosis of $\operatorname{IgA}$ to the apical surface. Here, we investigated a potential role for estrogen signaling in the regulation of $\mathrm{pIgR}$ expression in the lung to determine whether SIgA is decreased in bronchoalveolar lavage (BAL) fluid from women compared to men, and to determine whether pIgR expression in the murine lung is affected by estrogen signaling.

Methods: SIgA levels were measured in BAL fluid from 16 women and 7 men with mild COPD using a specific ELISA. pIgR mRNA and protein expression were quantified in lung lysates of estrogen receptor 1 and 2 null mice and controls by qPCR and western blotting, respectively. Pair-wise analyses were made using $t$-tests, with $\alpha=0.05$.

Results: SIgA levels were lower in BAL fluid from women with mild COPD compared to men with mild COPD (4.2 versus $6.9 \mathrm{mg} / \mathrm{ml}, P<.005)$. Both ER1 and ER2 null mice tended to have higher pIgR mRNA expression compared to wild-type female and male mice, suggesting that estrogen may suppress pIgR in the lung.

Conclusions: Estrogen-dependent signaling may suppress pIgR expression in the lung in a murine model. These findings may explain why younger women (i.e., premenopausal) are at higher risk for developing COPD.

\section{P-40: Probing Chemical Cues from the Ovarian Microenvironment}

Laura M. Sanchez (presenting author), Katherine E. Zink, and Joanna E. Burdette

Department of Medicinal Chemistry and Pharmacognosy, University of Illinois at Chicago

Background and Objective: In high-grade serous ovarian cancer, the 5-year survival rate is less than $10 \%$. A contributing factor to this low percentage is that because of a lack of diagnostic tests, most patients present with widespread disease at the time of diagnosis. Goal 2 of the ORWH Strategic Plan calls for "work toward devising minimally invasive technologies for rapid and accurate screening, diagnosis, and treatment of diseases." Our work seeks to exploit and expand our knowledge of imaging mass spectrometry (IMS) to probe the small-molecule signaling that governs early metastasis of ovarian cancer.

Methods: IMS is a powerful tool that allows for a chemical inventory defined by mass signals to be gathered from a biological sample along with spatial information. We hypothesized that cells derived from fallopian tubes that give rise to highgrade serous ovarian cancers express small-molecule signatures in order to rapidly colonize collagen surfaces that are exposed after ovulation. Our objectives were to adapt the IMS workflow to be compatible with agarose-embedded mammalian cell lines and to identify secreted small molecules from murine oviductal epithelial cells, the equivalent of the human fallopian tube, as they colonize the collagen.

Results: Our preliminary results demonstrated that our IMS platform can indeed be modified to be compatible with mammalian cell lines.

Conclusions: This method will allow us to explore new avenues in colonization and cellular signaling pathways that fallopian tube cells exploit to colonize the ovary microenvironment. The long-term goal is to disrupt these signals and block early metastasis of ovarian cancer.

\section{P-41: Readmission and Prolapse Recurrence: Quality Measures in Female Pelvic Reconstructive Surgery for Older Women}

Tatiana V.D. Sanses (presenting author), ${ }^{1}$ Jan M. Hanley, ${ }^{2}$ Peter Zhang, ${ }^{1}$ Holly E. Richter, ${ }^{3}$ Steve R. Gambert, ${ }^{1}$ and Chris S. Saigal ${ }^{4}$

${ }^{1}$ School of Medicine, University of Maryland; ${ }^{2}$ RAND Corporation; ${ }^{3}$ School of Medicine, University of Alabama at Birmingham; ${ }^{4}$ David Geffen School of Medicine, University of California, Los Angeles

Background and Objective: There are no quality measures in female pelvic reconstructive surgery specific to older women. We decided to evaluate 30-day inpatient readmission and 12month prolapse recurrence after abdominal and vaginal prolapse surgeries in older women.

Methods: This retrospective cohort study utilized the 20032010 Medicare files. It included women aged $\geq 65$ years who underwent abdominal sacrocolpopexy (ASC), vaginal sacrospinous ligament suspension (SSLS), or uterosacral ligament suspension (USLS) with at least 12-month follow-up. The primary outcome was 30-day inpatient readmission. Twelve-month prolapse recurrence was determined by the subject's needing surgical or conservative re-treatment for prolapse. Postoperative complications were evaluated. We used Pearson's chi-square and ANOVA tests to detect differences between procedures and logistic regression models to estimate odds ratios (ORs) for main outcomes.

Results: Thirty-day readmission was highest after ASC (7.4\%, compared with SSLS [3.5\%] and USLS [4.5\%], $P<.01$ ). Prolapse recurrence did not differ between the procedures. The ASC group had higher rates of gastrointestinal complications $(\mathrm{OR}=8.5 ; 95 \% \mathrm{CI}, 4.3,16.6)$ and surgical site infections $(\mathrm{OR}=1.8 ; 95 \% \mathrm{CI}, 1.2,2.8)$ than SSLS or USLS. The USLS group had the highest rates of bladder injury ( $4.9 \%$ vs. $3.2 \%$ [SSLS] and $2.4 \%$ [ASC], $P=.04)$ and ureter injury (3.7\% vs. $1.6 \%$ [SSLS] and $1.2 \%$ [ASC], $P<.01)$ as well as bladder dysfunction (13.7\% vs. $10.5 \%$ [SSLS] and $9.0 \%$ [ASC], $P=.02$ ).

Conclusions: We estimated national 30-day inpatient readmission and 12-month prolapse recurrence after abdominal and vaginal prolapse surgeries in older women. Our study provides information to create quality measures in female pelvic reconstructive surgery and data on surgical outcomes and complications specific to older women.

\section{P-42: The Role of Sex in Postoperative Cognitive and Functional Decline}

Katie J. Schenning (presenting author), ${ }^{1}$ Charles Murchison, ${ }^{2}$ Nora Mattek, ${ }^{2}$ Lisa Silbert, ${ }^{2}$ Jeffrey Kaye, ${ }^{2}$ and Joseph Quinn ${ }^{2}$ 
${ }^{1}$ Department of Anesthesiology \& Perioperative Medicine and ${ }^{2}$ Department of Neurology, Oregon Health \& Science University

Background and Objective: Older adults receive more than one-third of the over 40 million anesthetics delivered yearly in the United States, and these individuals are at the highest risk for postoperative cognitive decline. The role of sex in postoperative cognitive decline is unknown. We sought to determine whether exposure to general anesthesia (GA) and surgery in older adults is associated with an accelerated deterioration of cognition, function, and brain volumes, and whether this decline is more pronounced in women.

Methods: We performed a retrospective cohort analysis in 2 natural history studies of cognitive aging $(n=527)$. After controlling for age, years of education, and the Cumulative Illness Rating Scale, we used mixed-effects models to assess the relationship between exposure to GA/surgery and longitudinal change in measures of cognition, function, and brain volumes.

Results: Of the 527 participants, 182 underwent a total of 331 procedures under GA after enrollment in the study. While men exposed to surgery/GA experienced a more rapid rate of decline than their unexposed counterparts on the Mini-Mental State Examination (MMSE) $(P=0.009)$, on the Instrumental Activities of Daily Living Scale $(P=0.024)$, and on the Clinical Dementia Rating Scale Sum of Boxes (CDR-SOB, $P=0.027$ ), women exposed to surgery/GA experienced a significantly more rapid rate of deterioration than men in the following outcomes: MMSE $(P<.001)$, CDR $(P=.003)$, CDR-SOB $(P<.001)$, Activities of Daily Living Scale $(P<.001)$, Delayed Logical Memory $(P=.011)$, and ventricular volume $(P=.005)$.

Conclusions: In this retrospective cohort analysis, exposure to surgery/GA in older women was associated with more rapid rates of decline in measures of cognition, function, and brain volumes than older men.

\section{P-43: Reproductive Health Among Female Ebola Virus Disease Survivors}

John S. Schieffelin (presenting author), ${ }^{1}$ Fatima Kamara, ${ }^{5}$ Donald S. Grant, ${ }^{5}$ Jeffrey Shaffer, ${ }^{4}$ Marie Krousel-Wood, ${ }^{2}$ and Robert F. Garry ${ }^{3}$

${ }^{1}$ Department of Pediatrics, ${ }^{2}$ Department of Medicine, and ${ }^{3}$ Department of Microbiology and Immunology, School of Medicine, and ${ }^{4}$ Department of Biostatistics and Bioinformatics, School of Public Health and Tropical Medicine, Tulane University; ${ }^{5}$ Kenema Government Hospital, Sierra Leone

Background and Objective: The West African Ebola outbreak of 2013-2015 infected 28,610 people and caused 11,308 deaths. Reports from both the recent and past outbreaks have described myriad sequelae among survivors, including joint pain, headaches, vision problems, and depression. Amenorrhea has been occasionally reported. We sought to gain a better understanding of Ebola virus disease (EVD) sequelae, with an emphasis on women's reproductive health.

Methods: This study was carried out in Kenema District, Sierra Leone, approximately 16 months after the last reported case in the district. Eligible subjects had a documented history of EVD. All data was collected using standardized questionnaires and data collection forms.

Results: A total of 103 subjects were enrolled, of whom 69 $(67 \%)$ were female. Fifty-one (74\%) of the females were aged 15 years or older. Six percent of the females reported amenorrhea prior to having EVD, while $45 \%(P<.001)$ reported amenorrhea in the 12 months after recovering from EVD, and $40 \%(P<.001)$ reported amenorrhea in the past month. To determine the cause of amenorrhea, we performed a pilot study of 19 female EVD survivors. Two patients with amenorrhea were excluded because of a positive pregnancy test. FSH and prolactin levels of females with and without amenorrhea were compared. The mean FSH levels were 21.7 milli-international units $(\mathrm{mlU}) / \mathrm{ml}(2.4-54.4)$ and $11.9 \mathrm{mlU} / \mathrm{ml} \mathrm{(1.0-80.2)} \mathrm{for} \mathrm{the} \mathrm{amenorrheic} \mathrm{and} \mathrm{non-}$ amenorrheic groups, respectively, while prolactin levels were $7.7 \mathrm{ng} / \mathrm{ml}(4.7-9.8)$ and $26.0 \mathrm{ng} / \mathrm{ml}(2.7-112.1)$, respectively. No significant differences were noted.

Conclusions: More research is needed to identify gender differences in EVD survivors and the causes of morbidity to improve survivor care and treatment.

\section{P-44: Adapting an Evidence-based Recovery Intervention for Rural Women with Depression}

Claire Snell-Rood (presenting author), Lisa Ruble, and Nancy Schoenberg

\section{Department of Behavioral Science, University of Kentucky}

Background and Objective: The prevalence of depression among rural women is nearly 4 times the national average, and yet limited services and social barriers restrict access to treatment. Guided by formative research on depression among Appalachian women, we selected a culturally appropriate intervention-Wellness Recovery Action Planning (WRAP) to be implemented through community health workers (CHWs). Adaptations needed to be made to WRAP's delivery to encourage the participation of women whose poverty and depression are significant barriers to involvement. We aimed to identify strategies to adapt WRAP in order to encourage the participation and retention of rural women with depression.

Methods: In 4 focus groups with Appalachian women with depression $(n=10)$ and diverse health professionals $(n=10)$ who serve them, we examined how the evidence-based intervention should be adapted. Data were analyzed through content analysis and used to adapt WRAP. In follow-up interviews $(n=10)$, we solicited feedback on the adapted protocol to refine the adapted intervention.

Results: Consumers and providers recommended that health care professionals provide official referrals to WRAP and that the program be located in a health facility. The role of CHWs should be expanded to develop participants' trust, particularly through preintervention visits and contact outside of intervention sessions to maintain the relationship and provide logistic support. Women's retention in WRAP would be improved by offering incentives, ensuring confidentiality, and ensuring that materials are accessible for all literacy levels.

Conclusions: Adapting evidence-based mental health programs for rural women requires framing them as interventions for general health, ensuring facilitators can build meaningful relationships, and sustaining a welcoming environment.

\section{P-45: Association Between Sex and CD4 Cell Regeneration After Initiating Combination Antiretroviral Therapy in Resource-Limited Settings}

Kristen A. Stafford (presenting author) ${ }^{1}$ and Robert R. Redfield ${ }^{2}$ 
${ }^{1}$ Department of Epidemiology and Public Health and

${ }^{2}$ Institute of Human Virology, School of Medicine, University of Maryland

Background and Objective: Conflicting evidence exists regarding sex-based differences in the immunologic outcomes of combination antiretroviral treatment (cART). In resource-limited settings, sex-based differences in immune reconstitution are often attributed to higher adherence among women. We sought to estimate the association between sex and mean CD4 cell count over time by stratum of baseline CD 4 cell count among a group of HIV-infected patients with high adherence to cART.

Methods: Retrospective cohort study of adults followed for 5 years after beginning cART in 157 PEPFAR-funded clinics in 4 countries in sub-Saharan Africa. General linear regression models accounting for repeated measures were used to generate estimates.

Results: A total of 129,755 adults met study eligibility criteria, and 81,402 had an adherence level $\geq 95 \%$ in their first year of cART. In the baseline stratum of 0 to 200 cells, the mean difference in a comparison of women with men was +6 cells at baseline and +83 cells at 5 years, with an average mean difference of +61 . In the stratum of 201-350 cells there was no difference by sex at baseline, and at 5 years the mean difference was +62 cells (women over men), with an average mean difference of +58 cells. In the highest stratum ( $>350$ cells) women had +21 cells at baseline and +58 at 5 years, with an average mean difference of +85 cells. When subjects with $<95 \%$ adherence were included the mean differences were moderately attenuated.

Conclusions: Women demonstrated higher CD4 cell regeneration over time after starting cART when compared with men with having an equivalent number of CD4 cells at baseline.

\section{P-46: Adherence to the 2012 National Cervical Cancer Screening Guidelines at the Extremes of Age: A Retrospective Review}

Deanna Teoh (presenting author), ${ }^{1}$ Gretchen Hultman, ${ }^{2}$ Levi Downs, ${ }^{1}$ Melissa Geller, ${ }^{1}$ Eileen Harwood, ${ }^{3}$ Chap Le, ${ }^{4}$ Rachel Isaksson Vogel, ${ }^{1}$ Genevieve Melton-Meaux, ${ }^{2}$ and Shalini Kulasingam ${ }^{3}$

${ }^{1}$ Department of Obstetrics, Gynecology, and Women's Health; ${ }^{2}$ Department of Surgery; ${ }^{3}$ Division of Epidemiology \& Community Health, School of Public Health; and ${ }^{4}$ Department of Biostatistics, University of Minnesota

Background and Objective: To determine the prevalence of cervical cancer screening in women aged younger than 21 years or older than 65 years within the Fairview Health System.

Methods: The electronic health record was queried for all Pap tests performed in women $<21$ or $>65$ between September 1 , 2012, and August 31, 2014. Each chart was manually reviewed to determine the indication for the Pap test and whether the test was indicated based on the 2012 national cervical cancer screening guidelines.

Results: A total of 61,715 Pap tests were performed. Of these, $<1 \%(\mathrm{n}=512)$ were performed in women $<21$. Approximately $51 \%(\mathrm{n}=259)$ of tests in younger women were not indicated per the 2012 guidelines; if tests in patients within 6 months of their 21 st birthday were coded as not indicated, then $94 \%$ of tests of women $<21$ were not indicated. Reasons for non-indicated tests in this age group included routine health maintenance $(66.4 \%)$, pregnancy $(27 \%)$, and patient request $(7 \%)$. Approximately $2 \%$ $(n=1,327)$ of Pap tests were performed in women $>65$, of which $40 \%$ were not indicated. The reasons in this group for nonindicated tests were routine health maintenance $(88 \%)$, patient request $(7 \%)$, non-indicated follow-up of a previous abnormal test $(5 \%)$, and history of CIN2+> 20 years previously (.6\%). Of note, the most common reason for indicated testing in women $>65$ was inadequate documentation of previous screening $(56 \%)$.

Conclusions: Using conservative criteria, almost half of Pap tests performed in women who were aged either $<21$ or $>65$ years did not adhere to guidelines. Better education is needed regarding indications for Pap tests during pregnancy or following previous abnormal Pap tests.

\section{P-47: Vaginal Birth After Cesarean: Characteristics and Outcomes by U.S. Birth Setting}

Ellen Tilden (presenting author), ${ }^{1}$ Melissa Cheyney, ${ }^{4}$ Jeanne-Marie Guise, ${ }^{2}$ Cathy Emeis, ${ }^{1}$ Frances Biel, ${ }^{2}$ and Jonathan Snowden ${ }^{2,3}$

${ }^{1}$ School of Nursing, ${ }^{2}$ Department of Obstetrics and Gynecology, and ${ }^{3}$ School of Public Health, Oregon Health \& Science University; ${ }^{4}$ Department of Anthropology, Oregon State University

Background and Objective: In spite of 2010 clinical guidelines aimed at increasing U.S. rates of vaginal birth after cesarean (VBAC), VBAC rates remain low. Rates of U.S. outof-hospital birth increased between 2010 and 2014. Little is known about the characteristics and outcomes of women delivering out of hospital via VBAC. We sought to compare practice patterns and neonatal outcomes of VBAC in hospital, home, and birth center settings.

Methods: We used national birth certificate and linked infant death certificate data to identify VBAC deliveries between 2007 and 2010.

Results: Less than $9 \%$ of $1,138,813$ births were VBACs in any setting $(\mathrm{n}=109,970)$, with $96.5 \%$ of VBAC deliveries occurring in hospitals $(n=106,823)$. Women delivering in out-ofhospital settings had a significantly lower risk of intervention (labor induction 0.03 [95\% confidence interval (CI), 0.02, 0.05, $P<.001]$; labor augmentation 0.06 [95\% CI, 0.04, 0.07, $P<.001])$ compared with those born in hospital. Neonates born out of hospital had higher morbidity risk (Apgar $<7$ [adjusted odds ratio (AOR) 1.62 (95\% CI, 1.13, 2.82), $P<.001$ ], Apgar $<4$ [AOR $1.79(95 \% \mathrm{CI}, 0.42,2.48), P=0.014])$ and greater risk of neonatal seizures (AOR 7.29 [95\% CI, 2.5, 21.26], $P=0.003$ ) but equivalent mortality and risk of ventilator support (perinatal death, AOR 2.18 [95\% CI, 0.76-6.29], neonatal death, AOR 2.18 [95\% CI, 0.76, 6.29], infant death (AOR 1.58 [95\% CI, $0.72,3.49$ ], and ventilator support [AOR 1.36 (95\% CI, 0.75, 2.46)]) compared with those born in hospital.

Conclusions: VBAC rates are low in all settings. Equivalent perinatal mortality outcomes by setting may reflect a failure to disaggregate women who intended out-of-hospital VBAC but transferred to the hospital prior to birth. Lower intervention rates evident in out-of-hospital settings may reflect variations in practice patterns.

\section{P-48: Distinctive Reproductive and Metabolic Phenotypes in Peripubertal Girls at Risk for Polycystic Ovary Syndrome: PCOS Daughters Compared with Morbidly Obese Girls}


Laura C. Torchen (presenting author), ${ }^{1}$ Richard Legro, ${ }^{3}$ and Andrea Dunaif ${ }^{2}$

${ }^{1}$ Department of Pediatrics and ${ }^{2}$ Department of Medicine, Feinberg School of Medicine, Northwestern University; ${ }^{3}$ Department of Obstetrics and Gynecology, College of Medicine, Pennsylvania State University

Background and Objective: Polycystic ovary syndrome (PCOS) is a highly heritable disorder. Daughters of affected women (PCOS-d) develop increased testosterone (T) levels, a diagnostic criterion of PCOS, by early puberty. Obese girls (OBg) also have increased $T$ levels throughout puberty. Whether this increase in T production represents an early marker of PCOS in these girls is unknown. We aimed to compare reproductive and metabolic phenotypes in PCOS-d and OB-g, two groups of girls considered to be high risk for PCOS.

Methods: We studied 40 premenarchal PCOS-d, 25 OB-g, and 19 lean control girls of comparable age. An early-morning fasting blood sample was collected for measurement of antiMüllerian hormone (AMH), T, sex hormone binding globulin, estradiol, dehydroepiandrosterone, androstenedione, glucose, insulin, leptin, and adiponectin levels. Free T levels were calculated.

Results: PCOS-d and OB-g had similar increases in free $\mathrm{T}$ levels, compared with lean control girls. AMH levels differed, with significantly increased AMH in PCOS-d, compared with OB-g. Differences in metabolic variables were accounted for by differences in body mass index.

Conclusions: Although PCOS-d and OB-g have similarly increased free T, their AMH levels differ. The lower AMH levels in OB-g may suggest that they lack an increase in ovarian follicles, a key feature of the PCOS phenotype. Differences in these reproductive phenotypes may relate to the differing effects of PCOS susceptibility genes compared with the effect of obesity on development of the PCOS phenotype. Alternatively, early hyperandrogenemia in obese girls may not be an early marker of PCOS but rather an expected finding in peripubertal obesity.

\section{P-49: Sex Differences in Post-Traumatic Hippocampal Neurogenesis}

Laura E. Villasana (presenting author), ${ }^{1}$ Austin Peters, ${ }^{1,2}$ Gary L. Westbrook, ${ }^{3}$ and Eric Schnell ${ }^{1,4}$

${ }^{1}$ Department of Anesthesiology \& Perioperative Medicine, ${ }^{2}$ School of Medicine, and ${ }^{3}$ Vollum Institute, Oregon Health \& Science University; ${ }^{4}$ Portland Veterans Affairs Medical Center

Background and Objective: Studies suggest that women have poorer long-term cognitive outcomes than men following traumatic brain injury (TBI), including greater impairments in memory and mood. Both of these domains are sensitive to changes in the generation and integration of newborn neurons in the adult brain (neurogenesis). TBI increases neurogenesis within the hippocampus, a critical region for learning and memory, and this increase may influence cognitive recovery. The objective of this study was to determine whether there are sex differences in post-traumatic neurogenesis.

Methods: Adult male and female transgenic POMC-EGFP mice, which specifically express green fluorescent protein (GFP) in hippocampal adult-born neurons, underwent TBI using a controlled cortical impact or were sham treated. Two weeks later, neurogenesis was assessed by quantifying the density of GFP+ neurons within the hippocampus of each mouse. In a separate cohort of mice, proliferation of new cells in response to TBI was assessed using the mitotic marker bromodeoxyuridine (BrdU), which was injected intraperitoneally 2 days after TBI. The mice were sacrificed the following day and assessed for BrdU+ cells within the hippocampus.

Results: Compared with males, females had a greater density of GFP+ neurons 2 weeks after TBI, but there were no sex differences in the density of BrdU+ cells 3 days after TBI.

Conclusions: Our data suggest that female mice have a greater degree of post-traumatic neurogenesis than males. This may be due not to increases in proliferation but instead may involve other changes, such as increased neuronal survival. Regardlesss, we cannot rule out a potential bias towards greater neuronal differentiation in females.

\section{P-50: Mothers and Deployment: Understanding the Experiences and Support Needs of Military Mothers of Young Children}

Tova B. Walsh (presenting author)

\section{School of Social Work, University of Wisconsin}

Background and Objective: Women comprise a growing proportion of the U.S. military. Approximately $40 \%$ of female service members have children, and deployments have demanded long separations of mothers from their children. The strain of separation is particularly acute for mothers of very young children. This study aimed to better understand the deployment and reintegration experiences of service members who are mothers of young children.

Methods: Focus groups were conducted with women $(n=12)$ with 1 or more children aged $0-5$ years who served in the U.S. military and deployed to Iraq or Afghanistan. Focus group discussions explored experiences before, during, and after deployment; women's health, needs, and challenges faced at each stage; available support resources; and gaps in support. Discussions were audio recorded and transcribed. Thematic data analysis was conducted, drawing on principles of grounded theory, using QSR NVivo software.

Results: Common challenges experienced by mothers before, during, or after deployment included coming to terms with the impossibility of fully preparing young children and themselves for the experience of extended separation; worrying that things weren't going well at home and being unable to assess the situation or intervene; and readjusting after deployment to the competing demands of life at home as a mother of a young child. Mothers described reliance on family and friends for support, and they expressed discomfort accessing services geared toward male service members and their wives.

Conclusions: Mothers of young children face distinct challenges across the deployment cycle. Acquiring a better understanding of their experiences and support needs can inform tailored interventions to meet their needs.

\section{P-51: Degradation of the Vasoprotective Vascular Glycocalyx in Preeclampsia}

Tracey Weissgerber (presenting author), ${ }^{1}$ Natasa Milic, ${ }^{1,4}$ Elisabeth Codsi, ${ }^{2}$ Wendy White, ${ }^{2}$ Michelle Mielke, ${ }^{3}$ and Vesna Garovic ${ }^{1}$

${ }^{1}$ Division of Nephrology and Hypertension, ${ }^{2}$ Division of Maternal and Fetal Medicine, and ${ }^{3}$ Department 
of Epidemiology, Mayo Clinic; ${ }^{4}$ Department of Biostatistics, School of Medicine, University of Belgrade

Background and Objective: The vascular glycocalyx is a gel-like matrix of proteoglycans, glycoproteins, and plasma proteins positioned between the blood and the endothelium. Damage to this vasoprotective layer causes endothelial dysfunction. Glycocalyx degradation can be detected noninvasively by the greater penetration of red blood cells (RBCs) into the glycocalyx. We hypothesized that women with preeclampsia would show signs of glycocalyx degradation in sublingual microvessels.

Methods: Women with a normotensive pregnancy $(n=17)$ or preeclampsia $(n=9)$ were examined prior to delivery. Sidestream dark field imaging was used to record videos of RBCs flowing through the sublingual microvasculature. Glycocheck software was used to calculate the width of the portion of the glycocalyx that was permeable to RBCs and the percentage of vessels that were filled with RBCs. Repeated measures ANOVA was performed to examine the effects of preeclampsia after adjusting for vessel diameter.

Results: Women with preeclampsia had worse glycocalyx health when compared with normotensive pregnant women. The width of the portion of the glycocalyx that was permeable to RBCs was greater in women with preeclampsia ( 2.48 vs. 2.29 microns, $P=.005)$. The percentage of vessel segments that were filled with RBCs was lower in women with preeclampsia, compared with women with normotensive pregnancies (55\% vs. $60 \%, P=.005)$. Higher glycocalyx degradation and lower microvascular perfusion were observed in all vessel size categories.

Conclusions: Degradation of the vascular glycocalyx may contribute to the pathophysiology of preeclampsia by causing endothelial activation, microvascular injury, and reduced perfusion.

\section{P-52: Sex-Race Differences in Determinants of Adherence to Medication as Measured by Pharmacy Fill Among Older Insured Adults with Hypertension}

LaKeisha Williams (presenting author), ${ }^{1,2}$ Cara Joyce, ${ }^{3}$ Erin Peacock, ${ }^{2}$ Lydia Bazzano, ${ }^{4}$ Daniel Sarpong, ${ }^{1}$ Paul Muntner, ${ }^{5}$ and Marie Krousel-Wood ${ }^{2,3,6}$

${ }^{1}$ Division of Clinical and Administrative Sciences, College of Pharmacy, Xavier University of Louisiana; ${ }^{2}$ Department of Medicine, School of Medicine, ${ }^{3}$ Department of Biostatistics and Bioinformatics, and ${ }^{4}$ Department of Epidemiology, School of Public Health and Tropical Medicine, Tulane University; ${ }^{5}$ Department of Epidemiology, University of Alabama at Birmingham; ${ }^{6}$ Research Division, Ochsner Health System

Background and Objective: Sex and racial differences in adherence to antihypertensive medication have been reported; less is known about differences in determinants of low adherence among elderly sex-race subgroups. We sought to examine sex-race differences in determinants of low adherence to antihypertensive medication by using data on pharmacy fill among sex-race subgroups of older insured adults.

Methods: We employed a cross-sectional analysis using data from the Cohort Study of Medication Adherence among Older Adults (CoSMO, $n=2,122)$. Adherence to antihypertensive medication was calculated as the proportion of days covered (PDC); low adherence was considered a PDC less than $80 \%$.
Separate multivariate logistic regression models assessed factors associated with low adherence.

Results: Low adherence was identified in $22.9 \%$ of white women, $40.7 \%$ of black women, $26.3 \%$ of white men, and $37.2 \%$ of black men. Determinants associated with low adherence in white women were hypertension $<10$ years (odds ratio $[\mathrm{OR}]=1.74 ; 95 \%$ confidence interval $[\mathrm{CI}], 1.23,2.47)$, comorbidity score $\geq 2$ $(\mathrm{OR}=1.67 ; 95 \% \mathrm{CI}, 1.18,2.37)$, overall health care dissatisfaction $(\mathrm{OR}=2.37 ; 95 \% \mathrm{CI}, 1.08,5.20)$, and medication cost $(\mathrm{OR}=6.07$; $95 \%$ CI, 1.99, 18.54). Among black women, depressive symptoms $(\mathrm{OR}=1.76 ; 95 \% \mathrm{CI}, 1.00,3.08)$ and medication cost $(\mathrm{OR}=7.61$; $95 \%$ CI, 2.97, 19.46) were significant determinants of low adherence. Determinants associated with low adherence in white men were not being married $(\mathrm{OR}=1.55 ; 95 \% \mathrm{CI}, 1.02,2.34)$, medication cost $(\mathrm{OR}=3.85 ; 95 \% \mathrm{CI}, 1.48,10.01)$ and depressive symptoms $(\mathrm{OR}=1.88 ; 95 \% \mathrm{CI}, 1.03,3.43)$, while in black men, medication cost $(\mathrm{OR}=3.88 ; 95 \% \mathrm{CI}, .97,15.59)$ was marginally significant.

Conclusions: Sex-race differences in determinants of low adherence to antihypertensive medication were identified among sex-race subgroups of older insured adults.

\section{P-53: Toward the Identification of Endotypes of Maternal-Mediated Childhood Obesity}

ClarLynda Williams-DeVane (presenting author), ${ }^{1,2}$ Michele Josey, ${ }^{2}$ and Cathrine Hoyo ${ }^{3}$

${ }^{1}$ Biological and Biomedical Sciences Department and ${ }^{2}$ Bioinformatics, Genomics, and Computational Chemistry Core, Biomedical/Biotechnology Research Institute; and ${ }^{3}$ Department of Biological Science, Center for Human Health and the Environment, Epidemiology and Environmental Epigenomics Laboratory, North Carolina State University

Background and Objective: Given the burden of obesity on the U.S. population and economy, it is imperative that research efforts focus on decreasing the obesity rate. One such effort is through the early identification and understanding of risk in newborns. Differential epigenetic modifications are key to identifying early-life indicators of childhood obesity. Thus far, there has been limited success in identifying differential epigenetic modifications in newborn cord blood between normal and overweight/obese children because of a lack of statistical power and the use of broad indicators of disease. Here, we present a more targeted approach by investigating maternal-mediated childhood obesity, where the goal is to identify the mechanisms linking maternal obesity to obesity in the child using data from the Newborn Epigenetics STudy (NEST).

Methods: We applied the endotypes identification in complex diseases (EICD) iterative data integration framework to NEST data in the hope of finding more about the link between maternal and child obesity.

Results: We have developed classifications of BMI that are specific to sex and race and evaluated growth pattern modeling as an alternative to traditional BMI classifications. The new classifications will be used in a targeted analysis of 450,000 samples of epigenetic newborn cord blood to determine if we can better identify differential epigenetic modifications in the relationship between maternal obesity and obesity or overweight in the child.

Conclusions: These results will lead to better use of NEST epigenetic data as part of the EICD framework, leading to the identification of putative mechanisms and probable biomarkers of maternal-mediated childhood obesity and obesity in general. 


\section{P-54: Identification and Therapeutic Inhibition of Pathways Driving Resistance to Targeted Breast Cancer Therapies}

Kris C. Wood (presenting author), ${ }^{1}$ Grace R. Anderson, ${ }^{1}$ Suzanne E. Wardell, ${ }^{1}$ Jeff S. Jasper, ${ }^{1}$ Elizabeth M. Stein, ${ }^{1}$ Colin A. Martz, ${ }^{1}$ Holly M. Alley, ${ }^{1}$ Victoria Haney, ${ }^{1}$ Alexander Yllanes, ${ }^{1}$ Kimberly L. Blackwell, ${ }^{2}$ Shannon J. McCall, ${ }^{3}$ and Donald P. McDonnell ${ }^{1}$

${ }^{1}$ Department of Pharmacology and Cancer Biology, ${ }^{2}$ Department of Medicine, and ${ }^{3}$ Department of Pathology, Duke University

Background and Objective: Resistance to anticancer therapies is commonly driven by the activation of growth and survival signaling pathways that enable cancer cells to survive otherwise lethal drug treatments. By blocking these drug resistance pathways, it may be possible to improve the potency and durability of anticancer drugs. Unfortunately, for many drugs, including most targeted breast cancer therapies, the identities of potential resistance pathways are unknown. Using both functional genomic and pharmacological screening methods, this study sought to define the key pathways of resistance to selected targeted breast cancer therapies, then leverage this information to design combination therapies with improved efficacy.

Methods: We used a combination of pharmacological screening alongside pooled screens with an engineered library of pathway-activating mutant cDNAs to identify those pathways whose activation enhances the survival of breast cancer cells in the presence of clinically relevant targeted therapies. Mechanistic follow-up studies were performed using traditional biochemical and genetic methods, and translational follow-up studies were performed in orthotopic breast cancer xenograft models.

Results: These studies revealed a landscape of signaling pathways capable of driving resistance. Specifically, Notch1 activation drives resistance to the anti-estrogens tamoxifen and fulvestrant in ER+ breast cancers both in vitro and in vivo. Inhibition of this pathway reverses resistance in vivo, and Notch1 activity in a cohort of 458 patients predicted poor responses to upfront anti-estrogen treatment. Similarly, survival signaling through BCL-XL drives resistance to mTOR inhibition, and combined inhibition of mTOR and BCL-XL dramatically sensitizes $P I K 3 C A$ mutant breast cancers to $\mathrm{PI} 3 \mathrm{~K} / \mathrm{mTOR}$ pathway inhibition in vitro, in vivo, and in primary patient-derived tumors.

Conclusions: Systematic approaches can rapidly identify the pathways that drive clinical resistance, setting the stage for patient-directed studies. 


\section{BIRCWH Presentation Abstracts}

\section{O-1: Promoting Tissue Integration via Release of IL-4 from the Surface of Polypropylene Mesh}

Bryan N. Brown (presenting author), Daniel J. Hachim, and Samuel T. LoPresti

Department of Bioengineering, University of Pittsburgh

Background and Objective: Mesh implantation has been shown to improve anatomical outcomes in the repair of pelvic organ prolapse, but complications are observed in $>10 \%$ of cases. Complications are attributable, in part, to pro-inflammatory processes associated with the foreign body reaction mounted by the host. We sought to test the hypothesis that transient, earlystage shifts in macrophage polarization at the tissue-implant interface from a pro-inflammatory (M1) to an anti-inflammatory/ regulatory (M2) phenotype mitigate the foreign body reaction and improve implant integration downstream.

Methods: A nanometer-thickness coating capable of releasing IL-4 (an M2 polarizing cytokine) from an implant surface in a controlled manner was developed and evaluated in a rodent model.

Results: Results confirmed the presence of uniform loading of IL-4 throughout. The amount and release time of IL-4 were tunable based upon the number of coating bilayers. In vitro assays showed that implants coated with IL-4 promoted polarization to an M2 phenotype, demonstrating maintenance of IL-4 bioactivity following sterilization. In vivo studies showed that mice with IL-4 coated implants had increased percentages of M2 macrophages and decreased percentages of M1 macrophages at the tissue-implant interface during early stages of the host response. These changes were correlated with diminished formation of fibrotic capsule surrounding the implant and improved tissue integration downstream.

Conclusions: The results of this study demonstrate a versatile cytokine delivery system for shifting early-stage macrophage polarization at the tissue-implant interface and suggest that modulation of the innate immune reaction, rather than attempts to evade the immune system, may represent a preferred strategy for promoting the integration of biomaterials and the success of this modality.

\section{O-2: Impact of Sex and Menopausal Status on Episodic Memory Circuitry in Early Midlife}

Emily G. Jacobs (presenting author), ${ }^{1,2}$ Blair Weiss, ${ }^{1}$ Sue Whitfield-Gabrieli, ${ }^{3}$ Anne Klibanski, ${ }^{2,4}$ and Jill M. Goldstein ${ }^{1,2}$

${ }^{1}$ Brigham and Women's Hospital; ${ }^{2}$ Harvard Medical School, Harvard University; ${ }^{3}$ Massachusetts Institute of Technology;

${ }^{4}$ Massachusetts General Hospital

Background and Objective: Cognitive studies of the neuroscience of aging traditionally target participants aged 65 years or older. However, epidemiological surveys show that women report increased forgetfulness earlier in midlife, as they transition to menopause. In this population-based fMRI study, we stepped back by over a decade to characterize the changes in memory circuitry that occur in early midlife, as a function of sex and women's reproductive stage.

Methods: Participants ( $\mathrm{N}=200$, age range $45-55$ years) performed a verbal encoding task during fMRI scanning. Reproductive histories and serologic evaluations were used to determine menopausal status. Next, we examined the impact of sex and reproductive stage on functional connectivity across taskrelated brain regions.

Results: Findings revealed a pronounced impact of reproductive stage on task-evoked hippocampal responses, despite minimal difference in chronological age. Postmenopausal women showed enhanced bilateral hippocampal connectivity relative to premenopausal and perimenopausal women. Across women, lower $17 \beta$ estradiol concentrations were related to more-pronounced alterations in hippocampal connectivity and poorer performance on a subsequent memory retrieval task, strongly implicating sex steroids in the regulation of this circuitry. Subgroup analyses revealed that high-performing postmenopausal women (relative to low and middle performers) exhibited a pattern of brain activity akin to premenopausal women.

Conclusions: These findings underscore the importance of considering reproductive stage, not simply chronological age, to advance our understanding of memory circuitry function in the middle decades of life. In keeping with preclinical studies, these findings in humans suggest that the decline in ovarian estradiol production during menopause plays a significant role in shaping memory circuitry.

\section{O-3: Increased Fetal Inflammatory Gene Expression in Cell-Free Fetal RNA Is Associated with Earlier Gestational Age at Birth}

Neeta L. Vora (presenting author), ${ }^{1}$ Lisa Smeester, ${ }^{2}$ Kim Boggess, ${ }^{1}$ and Rebecca C. Fry ${ }^{2}$

${ }^{1}$ Department of Obstetrics \& Gynecology, University of North Carolina at Chapel Hill; ${ }^{2}$ Department of Environmental Sciences and Engineering, UNC Gillings School of Global Public Health

Background and Objective: There is emerging data that variants in the DNA of the fetus, not the mother, may be the trigger for some preterm births. The purpose of this study was to measure second-trimester fetal gene expression related to common preterm birth pathways (hypothalamic-pituitary axis, inflammatory, oxidative stress) using cell-free fetal RNA (cffRNA) extracted from second-trimester amniotic fluid supernatant in women who had spontaneous preterm birth (sPTB) and contrast it with women who delivered at term.

Methods: We performed a retrospective case-control study to compare fetal gene expression using cffRNA. CffRNA was extracted from amniotic fluid-derived stem cells of women with singletons who had second-trimester genetic amniocenteses. Twenty-one gravidas who had SPTB $(<34$ weeks) and 21 term controls ( $>37$ weeks) were matched for fetal sex, race, gestational age at amniocentesis, and medication exposure. Exclusion cri- 
teria included abnormal chromosomes or structural anomalies. CffRNA was extracted and hybridized to an 84-gene custom Nanostring platform including genes related to preterm birth pathways.

Results: Beta-2-microglobulin $(B 2 M)$ gene expression was significantly and negatively associated with gestational age (GA) at delivery $(P=.036, \beta=-.20)$. Differences in concentrations of nitric oxide synthase 1 and D-aspartate oxidase approached statistical significance in an analysis by GA at delivery $(P=.053 ; \beta=-.25$ and $P=.0504 ; \beta=-.31$, respectively).
Conclusions: Higher gene expression of $B 2 M$, a marker of the fetal inflammatory response, was noted in fetuses that ultimately had sPTB compared to those at term. Future research will determine whether alterations of fetal gene expression related to inflammation antedate clinical symptoms.

Financial support: Cefalo-Bowes Research Award from UNC-Chapel Hill Department of Obstetrics and Gynecology; RO3 HD080788; P42 ES005948; R01 ES019315; Building Interdisciplinary Research Careers in Women's Health K12 HD001441-16. 